\title{
L'accès à la justice en contexte numérique : l'information juridique par et pour les justiciables sur les médias sociaux ${ }^{+}$
}

\section{Alexandra Bahary-Dionne*}

Au Québec et au Canada, l'accès à la justice est une préoccupation croissante pour les milieux juridique, politique et universitaire. L'inaccessibilité financière des services juridiques engendre plusieurs problèmes pour un nombre important de justiciables, tout comme l'inaccessibilité cognitive de l'univers juridique. C'est dans ce contexte que les technologies de l'information et de la communication [TIC] occupent une place importante dans les discussions sur les manières de rendre la justice plus accessible. Elles auraient notamment le potentiel de fournir des ressources plus intelligibles et accessibles aux justiciables. Les médias sociaux en particulier pourraient démocratiser l'information juridique dans la mesure où ils sont faciles d'utilisation, rassemblent une diversité de contenus et sont de plus en plus ancrés dans les activités quotidiennes. Malgré l'intérêt que suscitent ces plateformes sur le plan de l'accès à la justice, leurs usages relatifs à l'information juridique restent à explorer pour la recherche en droit. Le présent article s'intéresse à ces plateformes, et en particulier au média social Facebook, qui a pour particularité de mettre en scène des internautes qui sont à la fois créateurs, récepteurs et relayeurs de contenus d'information juridique. Nous proposons finalement une typologie utile à la recherche sur le thème du droit et des médias sociaux.

Access to justice is an increasing concern for the legal, politic and academic spheres in Quebec and in Canada. Many problems are related to the financial inaccessibility of legal services for a considerable number of litigants, but also to the cognitive inaccessibility of the legal universe. In this light, information and communication technologies [ICT] make up an important part of the discussions about ways to make justice more accessible. More specifically, social media could democratize legal information insofar as they are easy to use, gather a diversity of content, and are more and more ingrained in daily activities. Despite the growing interest in these platforms in terms of access to justice, their uses pertaining to legal information are yet to be explored by legal researchers. This article hence focuses on these platforms and more specifically on Facebook, which allows users to simultaneously be creators, receptors and relators of legal information content. We finally introduce a typology useful to research oriented towards law and social media.

L'auteure tient à remercier Emmanuelle Bernheim, ainsi que les évaluateurs externes, pour leurs commentaires sur une version antérieure de ce texte.

* LL.B., Gr. Dip., étudiante à la maîtrise en droit à l’Université du Québec à Montréal 


\section{INTRODUCTION}

Au Québec et au Canada, l'accès à la justice est une préoccupation croissante pour les milieux juridique, politique et universitaire ${ }^{1}$. L'augmentation du nombre de justiciables non représentés par avocat ${ }^{2}$ [JNRA], tant dans les matières civiles et administratives que criminelles, constitue l'un des défís à l'ordre du jour ${ }^{3}$. Les études canadiennes démontrent que cette augmentation est due à l'incapacité des justiciables de payer pour des services juridiques ${ }^{4}$. À ce défi s'ajoute celui de l'incompréhension du langage juridique et du système de justice ${ }^{5}$. C'est dans ce contexte que la cyberjustice, qui désigne l'intégration des technologies de l'information et de la communication [TIC] au monde juridique ${ }^{6}$, occupe une place de plus en plus importante dans les discussions sur les manières de rendre la justice plus accessible. Plusieurs pensent qu'elle aurait le potentiel d'atténuer certains problèmes d'accès à la justice ${ }^{7}$, notamment en fournissant des ressources plus intelligibles et accessibles aux justiciables ${ }^{8}$. Les réflexions sur la cyberjustice ne se

1 Pierre-Claude Lafond, L'accès à la justice civile au Québec : portrait général, Cowansville, Yvon Blais, 2012 ; Pierre Noreau, Révolutionner la justice: constats, mutations et perspectives, Montréal, Éditions Thémis, 2010; Roderick Macdonald, « Access to Justice in Canada Today: Scope, Scale and Ambitions » dans Julia Bass, W A Bogart et Zemans, dir, Access to justice for a new century: the way forward, Toronto, Law Society of Upper Canada, 2005; Atteindre l'égalité devant la justice: une invitation à l'imagination et à l'action, Association du Barreau canadien, 2013, en ligne : $<\mathrm{https}$ //www.cba.org/CBA-Equal-Justice/Equal-Justice-Initiative/Reports?lang=fr-CA $>$ [Association du Barreau canadien]; M J Trebilcock, Lorne Mitchell Sossin et Anthony J Duggan, Middle Income Access to Justice, Toronto, University of Toronto Press, 2012.

2 À l'instar d'Emmanuelle Bernheim et de Richard-Alexandre Laniel, nous utiliserons le terme «justiciables non représentés » afin de mettre l'accent sur le fait qu'il s'agit rarement d'un choix ( «Un grain de sable dans l'engrenage du système juridique. Les justiciables non représentés : problème ou symptôme? » (2013) 31:1 Windsor B Access Justice 45) [Bernheim et Laniel]. À la question de savoir s'ils préfèreraient agir seuls ou être représentés par un avocat à la cour, $88,1 \%$ des Québécois affirment qu'ils souhaiteraient être représentés par un avocat (Projet Accès au droit et à la justice), « Justice pour tous », en ligne : http://adaj.ca/justicepourtous/sondage (consulté le 12 novembre 2018).

3 En 2010, 25 \% des demandes de pourvoi à la Cour suprême étaient le fait d'un JNRA (Cour suprême du Canada, Budget des dépenses 2010-2011 - Un rapport sur les plans et les priorités, Ottawa, 2010, en ligne: https://www.tbssct.gc.ca/rpp/2010-2011/inst/jsc/jsc-fra.pdf). Jusqu'à $40 \%$ des affaires en matière criminelle dans les cours provinciales impliqueraient un JNRA (ministère de la Justice du Canada, Étude nationale sur les adultes non représentés accusés devant les cours criminelles provinciales - Partie 1, Ottawa, 2002 à la p 17.) En matière civile à la Cour supérieure, $31 \%$ des dossiers comportent au moins un JNRA (François Rolland, «La justice du $21^{\mathrm{e}}$ siècle », Accès à la justice - Effets concrets sur la pratique du droit, Congrès du Barreau du Québec, Gatineau, 4 juin 2011).

4 Julie Macfarlane, The National Self-Represented Litigants Project: Identifying and Meeting the Needs of Self-Represented Litigants, Final Report, Rapport de recherche présenté aux fondations du droit de l'Ontario, de l'Alberta et de la ColombieBritannique, Canadian Forum on Civil Justice, 2013, en ligne : $<$ http://www.lsuc.on.ca/uploadedFiles/For_the_Public/About_the_Law_Society/Convocation_Decisions/2014/Selfrepresented_project.pdf $>$ [Macfarlane]; Rachel Birnbaum, Nicholas Bala et Lorne Bertrand, "The Rise of Self Representation in Canada's Family Court: the Complex Picture Revealed in Surveys of Judges, Lawyers and Litigants » (2013) 91 Rev Barreau Can.

5 Pierre-Claude Lafond, L'accès à la justice civile au Québec : portrait général, Cowansville, Yvon Blais, 2012; Pierre Noreau, Révolutionner la justice : constats, mutations et perspectives, Montréal, Éditions Thémis, 2010.

6 Karim Benyekhlef et al, dir, eAccess to justice, Ottawa, University of Ottawa Press, 2016.

7 Dory Reiling, Technology for Justice: How Information Technology Can Support Judicial Reform, coll Law, governance, and development Dissertations, Amsterdam, Leiden University Press, 2009.

8 James E Cabral et al, « Using technology to enhance access to justice » (2012) 26 Harv JL Tech 241 ; Jean Hoefer Toal et al, Future Trends in State Courts 2007 Increasing Access to Justice for the Self-Represented Through Web Technologies, 
limitent pas aux possibilités de numérisation des institutions judiciaires : pour certaines auteures, elles doivent s'élargir aux « processus juridiques informels » qui offrent de nouvelles manières d'accéder à l'information, notamment par le biais des médias sociaux ${ }^{9}$. Si l'essor des TIC a permis de faciliter l'accès à l'information dans divers domaines ${ }^{10}$, plusieurs études se sont intéressées plus spécifiquement à l'utilisation des médias sociaux à des fins de partage et d'échange d'information ${ }^{11}$. Cet engouement particulier s'explique par le potentiel lié à l'émergence du «web social », qui caractérise les récents développements d'Internet permettant de «favoriser des pratiques de collaboration et de partage d'information entre internautes ${ }^{12}$ et qui met ainsi en scène des usagers actifs plutôt que des consommateurs d'information passifs. Dans le domaine juridique, certains croient d'ailleurs que ce sont les médias sociaux qui pourront démocratiser l'information juridique en atténuant les barrières financières et informationnelles existantes pour les $\mathrm{JNRA}^{13}$. Le potentiel spécifique aux médias sociaux serait aussi lié au fait que, contrairement à d'autres dispositifs sociotechniques, ils sont de plus en plus ancrés dans les activités du quotidien ${ }^{14}$. En 2016, au Québec, plus de $67 \%$ des adultes avaient un compte actif sur un ou plusieurs médias sociaux et $64 \%$ utilisaient Facebook ${ }^{15}$.

Cette rencontre entre le droit et les médias sociaux amène en elle-même de nouveaux questionnements pour la recherche en droit. Malgré l'intérêt que cette rencontre suscite en matière d'accès à la justice, les études empiriques en la matière restent rares. Plus particulièrement, les usages des médias sociaux à des fins de partage d'information juridique restent à documenter ${ }^{16}$. On croit pourtant que les personnes n'ayant pas accès à des services juridiques sont particulièrement susceptibles d'utiliser les médias sociaux ${ }^{17}$, ce qui laisse supposer qu'une meilleure compréhension de ces usages pourrait contribuer à éclairer le milieu juridique sur les besoins de ces justiciables.

États-Unis, National Center for State Courts, 2007, en ligne : $<$ http://ndcrc.org/sites/default/files/future_trends_2007.pdf $>$.

9 Suzanne Bouclin et Marie-Andrée Denis-Boileau, « La cyberjustice comme réponse aux besoins juridiques des personnes itinérantes : son potentiel et ses embûches » (2013) 31:1 Windsor B Access Just 23 à la p 35 [Bouclin et Denis-Boileau].

10 Frank Webster, Theories of the Information Society, $4^{\mathrm{e}}$ éd, Londres, Routledge, 2014.

11 Florence Millerand, Serge Proulx et Julien Rueff, Web social : Mutation de la communication, Québec, Les Presses de l’Université du Québec, 2010.

12 Florence Millerand, «Les TIC dans les sciences. Pistes de problématiques pour l'étude des dynamiques d'usage et d'innovation technique » dans Christian Agbobli, dir, Quelle communication pour quel changement? : les dessous du changement social, Québec, Presses de l’Université du Québec, 2009.

13 Cassandra Burke Robertson, « The Facebook Disruption: How Social Media May Transform Civil Litigation and Facilitate Access to Justice » (2012) 65 Ark Rev 75 [Robertson].

14 Tim Highfield, Social Media and Everyday Politics, Cambridge, RU, Polity, 2016.

15 «Médias sociaux et économie de partage en ligne au Québec », CEFRIO NETendances 2016 7:9 (2017), en ligne : CEFRIO NETendances $2016<$ https://cefrio.qc.ca/media/1228/netendances_2016-medias-sociaux-et-economie-departage-en-ligne-au-quebec.pdf $>$.

16 À titre indicatif, une recherche avec les mots-clés «social media» et «legal information»a donné 762 résultats sur HeinOnline le 6 mars 2018. La plupart des résultats portent sur la transformation de la profession juridique. Les articles trouvés ne comportent pas de résultats de recherche empirique. Une recherche de Caroline Paetcher dans le domaine des sciences de l'éducation s'intéresse toutefois au partage d'expériences en lien avec le divorce sur un forum en ligne, quoique l'accent ne soit pas spécifiquement mis sur l'information juridique, ce qui laisse supposer que ce dernier aspect reste à explorer (« Online Learning in Divorce: How the Formation of a Community of Practice in a Divorce Support Website Enables and Inhibits Learning » (2012) 9:4 E-Learn Digit Media 392).

17 Lafond, supra note 5. 
Faisant état d'une recherche en cours sur le thème des pratiques informationnelles des justiciables sur les médias sociaux, le présent article s'intéresse en particulier au média social Facebook qui a pour particularité de mettre en scène des internautes qui sont à la fois créateurs, récepteurs et relayeurs d'information juridique. Quelles sont les particularités des médias sociaux au regard de l'accès à l'information juridique et dans quels types d'espaces plus spécifiques peut-on retrouver ces pratiques informationnelles ? Nous proposons d'abord de faire un état des lieux du contexte socioéconomique dans lequel ces pratiques informationnelles émergent, soit les différentes barrières d'accès aux services juridiques. Nous aborderons ensuite les enjeux liés à l'émergence des TIC dans l'univers juridique, en particulier les plateformes d'information juridique, tant sur le plan de leur conception que de leur réception par les internautes. Nous discuterons ensuite du rôle spécifique des médias sociaux en matière d'information juridique : les internautes y sont à la fois émetteurs et récepteurs de contenus. Nous aborderons par la suite les approches théoriques et méthodologiques qui pourraient permettent de documenter ce phénomène, ainsi que leur utilité pour comprendre les réalités des individus ayant des besoins de nature juridique dans le contexte socionumérique actuel. Pour finir, nous présenterons une typologie des différentes pages et différents groupes Facebook utilisés pour échanger de l'information juridique dans le contexte québécois. Cette classification permet de déterminer si et dans quels contextes d'échanges ces pratiques informationnelles ont lieu sur ce média social. Cette typologie distingue 1) les pages et les groupes dont le contenu vise en tout ou en partie l'information juridique ; 2) les groupes qui rassemblent des internautes vivant une situation commune dans le système de justice, comme un recours collectif et 3) les groupes dont l'objectif, selon leur description, n'est pas de partager de l'information juridique, mais où l'on peut retrouver des questions qui y sont reliées de manière ponctuelle en raison de la grande taille du groupe. Cette typologie permet de constater que les pratiques informationnelles en matière juridique sont extrêmement variées au sein des médias sociaux : elles peuvent viser des groupes aux objectifs très différents, avec un nombre de membres et des niveaux d'interaction très variés ; plusieurs peuvent être accessibles à tout internaute. Une partie de ces contenus se retrouve d'ailleurs dans des groupes qui n'ont aucun lien apparent avec le droit étatique, selon leur description.

\section{L'ACCÈS AUX SERVICES JURIDIQUES : DES BARRIÈRES FINANCIÈRES ET INFORMATIONNELLES}

Actuellement, l'un des enjeux principaux du système de justice est l'accès aux services d'un avocat ${ }^{18}$. Au Québec, pour la période 2014-2015, près de $38 \%$ des justiciables en matière familiale n'étaient pas représentés par un avocat ${ }^{19}$. Cette situation est fortement évoquée dans les publications professionnelles et scientifiques québécoises, mais demeure peu documentée sur le plan empirique, contrairement aux autres provinces canadiennes et aux États-Unis ${ }^{20}$. Le ministère de la Justice a identifié comme causes d'une part, les coûts de la justice et d'autre part, le bas niveau des seuils d'admissibilité aux services d'aide

18 Macdonald, supra note 1.

19 Cette proportion est de $55 \%$ pour les autres principaux domaines en matière civile : ministère de la Justice du Québec, Plan stratégique 2015-2020, Bibliothèques et Archives nationales du Québec, 2016 à la p 14, en ligne :

$<$ https://www.justice.gouv.qc.ca/fileadmin/user_upload/contenu/documents/Fr_francais_/centredoc/publications/minist ere/plans-strategiques/plan-strat1520.pdf $>$.

20 Bernheim et Laniel, supra note 3. 
juridique ${ }^{21}$. Les services juridiques sont inaccessibles pour un nombre croissant de justiciables, selon Pierre-Claude Lafond :

sur le chapitre des honoraires, en pratique, seules les personnes très fortunées, les grandes entreprises, les organisations gouvernementales et les personnes admissibles à l'aide juridique sont capables de s'offrir le luxe d'une action en justice ${ }^{22}$.

Au Québec, plus de 69,3\% de la population québécoise estime ne pas avoir les moyens d'aller devant les tribunaux ${ }^{23}$. Une enquête de terrain menée en Ontario, en Alberta et en Colombie-Britannique révèle que $90 \%$ des JNRA sont non représentés pour des raisons financières ${ }^{24}$.

Certaines recherches démontrent que les barrières à l'accès à la justice ne sont pas uniquement financières, mais aussi informationnelles, géographiques et psychologiques ${ }^{25}$. Au Canada, le manque de connaissances au sujet du système de justice a été identifié comme un frein important pour ester en justice $^{26}$. Cela limiterait l'accès à un avocat, mais aussi à 1'information juridique ${ }^{27}$. Les personnes appartenant à un groupe marginalisé pour diverses raisons (analphabétisme, situation d'itinérance, handicap) vivraient ces barrières de manière exacerbée ${ }^{28}$. Certains de ces groupes risquent d'ailleurs de connaître plusieurs problèmes juridiques à la fois ${ }^{29}$. Selon Jane Bailey, Jacquelyn Burkell et Graham Reynolds :

Self-represented litigants in Canada are disproportionately likely to have lower income and education, and to live with social barriers including physical and mental differences, and language and cultural barriers; furthermore, they often live in rural areas remote from physical court and legal services ${ }^{30}$.

21 Québec, ministère de la Justice, Rapport de gestion 2009-2010, Québec, 2010, à la p 37.

22 Lafond, supra note 1 à la $\mathrm{p} 122$. Quant au régime d'aide juridique, il « règle le problème des plus pauvres, ce qui n'est pas négligeable, mais ne tient pas compte des pauvres qui gagnent le salaire minimum ou qui font partie de la classe moyenne. » (Ibid, à la p 120). En janvier 2016, les seuils d'admissibilité à l'aide juridique ont toutefois été haussés pour qu'une personne seule travaillant 35 heures par semaine au salaire minimum y ait droit. En mai 2017, ils ont été arrimés à la hausse avec le salaire minimum (« Hausse des seuils d'admissibilité à l'aide juridique depuis le 31 mai 2017 » (6 mai 2017), en ligne: Services juridiques communautaires de Pointe-Saint-Charles et Petite-Bourgogne $<$ http://www.servicesjuridiques.org/hausse-des-seuils-dadmissibilite-a-laide-juridique-depuis-le-31-mai-2017/> (consulté le 17 juillet 2017).)

23 INFormation, Recherche et Analyse de la Société inc., Rapport : Enquête sur le sentiment d'accès et la perception de la justice au Québec, réalisé pour le ministère de la Justice du Québec, avril 2016 à la p 18.

24 Macfarlane, supra note 4 aux pp 39 et 48.

25 Association du Barreau canadien, supra note 1.

26 Ibid à la $\mathrm{p} 34$.

27 Jena McGill, Suzanne Bouclin et Amy Salyzyn, «Mobile and Web-Based Legal Apps: Opportunities, Risks and Information Gaps » (2017) 15 Can J Law Technol 230.

28 Bouclin et Denis-Boileau, supra note 9.

29 Ibid

30 Bailey, Burkell et Reynolds, « Access to Justice for All: Towards an Expansive Vision of Justice and Technology » (2013) 31 Windsor B Access Just 181 à la p 169. 
Une «vision élargie» de l'accès à la justice, qui ne se cantonne pas à la représentation ou à l'administration des tribunaux, est donc nécessaire pour appréhender les multiples inégalités dans l'accès au système de justice :

The expansive vision $[\ldots]$ recognizes that socioeconomic and other structural differences among citizens affect their respective abilities to benefit both from the justice system itself and from initiatives designed to improve access to justice. ${ }^{31}$

Parmi ces initiatives figurent les possibilités que la technologie offre pour atténuer ces barrières d'accès ${ }^{32}$. Ron Dolin nous met d'ailleurs en garde contre le scepticisme à l'égard des technologies face à l'augmentation du nombre de JNRA :

The issue is not whether technology can compare with a given attorney, or even the worst tolerable one. [...] Where the alternative for a particular person is no lawyer due to a lack of personal or societal resources, then the technology needs to be compared with that individual going it alone. ${ }^{33}$

Il est dès lors d'autant plus pertinent d'explorer les usages des TIC - et plus spécifiquement des médias sociaux - que font les justiciables privés d'accès à des services juridiques, en prenant soin d'intégrer une vision élargie de l'accès à la justice qui tienne compte des enjeux propres à l'utilisation de ces technologies.

\section{LA CYBERJUSTICE : POSSIBILITÉS ET DÉFIS POUR L'ACCÈS À L'INFORMATION JURIDIQUE}

Les liens entre le droit et la technologie attirent l'attention de nombreux chercheurs depuis plus d'une décennie. Les principaux domaines de recherche portant sur ces liens s'intéressent au cadre juridique de l'utilisation des TIC dans le contexte de la mondialisation ${ }^{34}$, à la règlementation du cyberespace, notamment en ce qui a trait à la propriété intellectuelle ${ }^{35}$, mais aussi aux changements amenés par les TIC sur l'offre des services juridiques ${ }^{36}$ et sur le développement des connaissances juridiques ${ }^{37}$. L'ensemble de ces travaux témoignent d'un intérêt croissant pour le champ de la cyberjustice. Selon Karim Benyekhlef, ce terme désigne à la fois l'intégration des TIC dans les modes de règlements de litiges

$31 \quad$ Ibid à la p 182.

32 McGill, Bouclin et Salyzyn, supra note 27.

33 Ron Dolin, «UPL, Technology, and Access to Justice» (30 avril 2015), en ligne : Radical Concepts $<$ http://radicalconcepts.com/285/upl-technology-and-access-to-justice/>.

34 Irene Maria Portela et Maria Manuela Cruz-Cunha, dir, ICT Law, Protection and Access Rights: Global Approaches and Issues, Hershey PA, Information Science Reference, 2010.

35 Lawrence Lessig, Code and Other Laws of Cyberspace, Version 2.0, New York, Basic Books, 2006.

36 Ethan Katsh, Law in a Digital World, New York, Oxford University Press, 1995; Richard Susskind, The End of Lawyers? Rethinking the Nature of Legal Services, Oxford, Oxford University Press, 2010.

37 Daniel Poulin, «La démocratisation de l'accès au droit: nouveaux acteurs et nouvelles technologies » dans Claire Monville, dir, Variations sur le droit de la société de l'information, Bruxelles, Cahiers du CRID, $2011,27$. 
judiciaires et extrajudiciaires et la mise en réseau par le numérique des différentes parties prenantes dans les affaires judiciaires ${ }^{38}$. La cyberjustice vise ainsi la modernisation, la mise en réseau et la réingénierie des procédures judiciaires permettant la circulation des données entre les différents acteurs d'une chaine d'information ${ }^{39}$, mais aussi le partage d'information juridique ${ }^{40}$. Pour certaines chercheuses, la cyberjustice s'exerce non seulement au profit des professionnels du droit, mais aussi des justiciables «principalement en intégrant les TIC dans le processus judiciaire et en mettant à la disposition des gens des outils de simplification et de vulgarisation des procédures judiciaires et du droit $»^{4}$. Elles évoquent la mise en marché de plateformes numériques, incluant des applications juridiques web ou mobiles, pour faciliter l'accès aux services et à l'information disponibles. Les plateformes numériques seraient aussi utiles pour atténuer les barrières financières, physiques et géographiques de l'accès à la justice ${ }^{42}$.

L'appropriation de ces plateformes par les justiciables, en particulier les JNRA, comporte aussi des limites et des défis. Certains auteurs soulignent l'importance de concevoir des technologies qui n'exacerbent pas le manque d'accès à la justice pour les destinataires visés par ces initiatives ${ }^{43}$. Pour intégrer les TIC, il faudrait d'abord considérer les barrières inhérentes à l'accès à la technologie ${ }^{44}$, sans quoi il y aurait un risque de fracture numérique. Une telle fracture encouragerait un système à deux vitesses entre les justiciables ayant accès à ces dispositifs et ceux qui n'y ont pas accès ${ }^{45}$.

38 Supra note 8 à la p 1.

39 Karim Benyekhlef et Fabien Gélinas, Le règlement en ligne des conflits : enjeux de la cyberjustice, Paris, Romillat, 2003. Voir aussi Harold Epineuse, Lignes directrices sur la conduite du changement vers la cyberjustice : Bilan des dispositifs déployés et synthèse de bonnes pratiques, CEPEJ (2016)13É, Strasbourg, CEPEJ, 2016.

40 Susskind, supra note 38. L'auteur croit qu'il est nécessaire de concevoir une politique publique sur l'accessibilité des sources primaires du droit et de l'information juridique en ligne.

41 McGill, Bouclin et Salyzyn, supra note 27 aux pp 11 et 44.

42 Bailey, Burkell et Reynolds, supra note 30. Cet aspect est particulièrement intéressant pour les populations rurales : Cabral et al., supra note 8 à la $\mathrm{p} 261$. Cela dit, l'accès à un service Internet haute vitesse demeure un enjeu important pour les régions du Québec et du Canada (voir Maxime Poiré, "Internet haute vitesse : vers la fin de la discrimination numérique?», Radio-Canada (4 décembre 2017), en ligne: Radio-Canada <https://ici.radiocanada.ca/nouvelle/1070463/internet-haute-vitesse-regions-discrimination-numerique>.

43 Bailey, Burkell et Reynolds, supra note 30 à la p 198. Par exemple en utilisant des interfaces peu conviviales.

44 Patricia Hughes, « Advancing Access to Justice through Generic Solutions: The Risk of Perpetuating Exclusion » (2013) 31 Windsor B Access Just 1.

45 Cabral et al, supra note 8 à la p 265. La présence en ligne ne présuppose pas, en principe, un niveau de revenu ou de scolarité particulier. Or, il existe encore des disparités dans l'accès à Internet en fonction du niveau de revenu et de scolarité - quoique cet écart se soit amenuisé au cours des dernières années. En 2016, 68,5\% des ménages ayant un revenu dans le premier quartile étaient branchés à Internet (59,5\% en 2012), par rapport à 89,9 à 98,4\% pour les trois autres quartiles (79,7 à 96,0 \% en 2012) : Institut de la statistique du Québec, Enquête québécoise sur l'accès des ménages à Internet 2012 et 2016, Bibliothèque et Archives nationales du Québec, 2017. Au total, 88,2\% des ménages québécois ont accès à Internet en 2016 (81,6\% en 2012). L'écart selon le niveau de scolarité est aussi très marqué : il est respectivement de 95,2 et 97,8 \% par rapport à 52,1\% pour les ménages sans diplôme (44,1\% en 2012) (Ibid; Marianne Bernier, «L'accès des ménages à Internet en $2012 »$, Science, technologie et innovation en bref, novembre 2017, Institut de la statistique du Québec, en ligne $<$ http://www.stat.gouv.qc.ca/statistiques/science-technologie-innovation/utilisation-internet/menages-

individus/cahier-metho-acces-menage-internet.pdf $>$ ). Toutefois, si l'âge a une forte incidence sur l'utilisation des médias sociaux, les niveaux de revenu et de scolarité n'ont pas d'effet significatif alors qu'un peu plus de 4 internautes sur 5 utilisent les réseaux sociaux: Les médias sociaux, au cœur du quotidien des Québécois, 5, coll NETendances 2014, CEFRIO, 2014 à la p 4, en ligne : <http://www.cefrio.qc.ca/netendances/medias-sociaux-coeur-quebecois/>. En somme, si l'accès à Internet constitue une première barrière pour les personnes à faible revenu ou peu scolarisées, celles-ci ne sont 
Or, la question de l'accès se pose au-delà de sa dimension matérielle : les chercheurs qui s'intéressent aux usages des TIC doivent appréhender la thématique de l'inégalité d'accès « dans l'appropriation de la culture numérique ${ }^{46}$. Des auteurs ayant exploré les usages d'Internet par les jeunes pour répondre à des problèmes de nature juridique mettent ainsi en lumière une deuxième fracture numérique « qui va au-delà de l'accès pour explorer les usages et les résultats de ces usages $»^{47}$. L'étendue de cette fracture, qui consacre la volonté et la capacité d'utiliser Internet à des fins informationnelles, reste à documenter. Mais surtout, la question à savoir comment les internautes utilisent les plateformes numériques pour obtenir de l'information juridique demeure peu explorée, notamment parce qu'elle pose plusieurs défis méthodologiques. Catrina Denvir soulève par exemple la difficulté de comprendre ce processus de recherche d'information en dehors de situations de test, et donc pour répondre à des problèmes juridiques réels ${ }^{48}$.

Ces préoccupations impliquent de mieux définir la notion d'information juridique. Le sociologue Theodore Roszak appelle à une appréciation qualitative de l'information en distinguant la notion de données de celles de sagesse, de connaissance et d'information. Si les données représentent des éléments bruts, la connaissance permet de discriminer parmi ces données, et le savoir permet d'organiser l'information. L'information, quant à elle, doit être productrice de sens : elle doit avoir un sujet et donner une signification à quelque chose ou à quelqu'un ${ }^{49}$. Aux fins du présent article, nous définissons l'information juridique comme un élément qui donne une signification au droit ou au rapport de celui-ci avec la situation personnelle de l'internaute. À cet égard, comme le précise Lafond, l'accès matériel à l'information juridique ne présuppose pas de l'habileté à traiter cette information: «Trop souvent, l'information se veut trop largement accessible et le citoyen est incapable de faire le tri pour retenir celle qui est pertinente à son cas $\rangle^{50}$. L'abondance de l'information en ligne et la difficulté de discriminer parmi cette information peuvent faire en sorte que les internautes s'y perdent ${ }^{51}$. Les participants à une étude québécoise sur les besoins et défis liés à l'autoreprésentation ont d'ailleurs rapporté ce problème dans leur processus de recherche d'information ${ }^{52}$. En matière juridique en particulier, la littératie, qui consiste en

pas susceptibles de moins utiliser les médias sociaux une fois qu'elles y ont accès.

46 Serge Proulx, «L'irruption des médias sociaux : enjeux éthiques et politiques » Serge Proulx, Mélanie Millette et Lorna Heaton, dir, Médias sociaux : Enjeux pour la communication, Québec, Presses de l'Université du Québec, 2012 à la p 11.

47 Catrina Denvir, Nigel J Balmer et Pascoe Pleasence, « Surfing the web - Recreation or resource? Exploring how young people in the UK use the Internet as an advice portal for problems with a legal dimension » (2011) 23:1 Interact Comput 96, DOI : 10.1016/j.intcom.2010.10.004. (Notre traduction)

48 Catrina Denvir, « Online and in the know? Public legal education, young people and the Internet » (2016) 92-93 Comput Educ 204, DOI : 10.1016/j.compedu.2015.10.003.

49 Theodore Roszak, The Cult of Information: A Neo-Luddite Treatise on High-Tech, Artificial Intelligence, and the True Art of Thinking, $2^{\mathrm{e}}$ éd, Berkeley, University of California Press, 1994.

50 Lafond, supra note 1 à la $\mathrm{p} 78$.

51 Le sociologue de l'information Frank Webster distingue d'ailleurs le fait d'avoir de l'information et d'être informé : « While being informed requires that one has information, it is a much grander condition than having access to masses of information »: Supra note 10 à la p 32.

52 Équipe de recherche du chantier 1 - Autoreprésentation et plaideur citoyen, « La force du suivi personnalisé pour les personnes autoreprésentées », Rapport de recherche sur la Clinique juridique du Mile End, Montréal, février 2018. Si cette recherche portait plus généralement sur les expériences des usagers d'une clinique juridique en tant que JNRA, ces résultats démontrent le besoin de documenter de manière plus approfondie le processus de recherche d'information en ligne par ces JNRA [« La force du suivi personnalisé pour les personnes autoreprésentées »]. 
compétences variées de lecture et d'écriture ${ }^{53}$, constitue aussi un enjeu que Patricia Hugues décrit avec éloquence :

Individuals using information, however acquired, must be able to read it, understand it and apply it to their own situation. Each of these tasks requires an increasing level of literacy. Yet a significant minority of people lack the required literacy even to understand the information, particularly since it is often difficile to avoid legal terminology and the information can quickly become complex ${ }^{54}$.

L'information disponible en ligne doit donc être réellement accessible et intelligible pour les internautes ${ }^{55}$, ce qui soulève l'importance pour la recherche de distinguer l'étape de la conception de l'information juridique de celle de sa réception par l'auditoire. Or, la question de l'émergence et de l'appropriation de l'information juridique en ligne en suppose une plus spécifique: qu'en est-il des plateformes caractéristiques du web social, où les internautes sont à la fois les émetteurs et les récepteurs des contenus d'information juridique?

\section{INFORMATION JURIDIQUE ET MÉDIAS SOCIAUX : DES SAVOIRS JURIDIQUES COLLECTIFS ?}

Dès sa genèse, Internet réunit « sur la même interface les outils de l'échange interpersonnel de ceux de la communication de masse $»^{56}$. Le sociologue Manuel Castells parle de « médias de masse individuels $»^{57}$, qui instaurent ainsi «un nouveau type de relation entre la sphère de la conversation et celle de l'information $»^{58}$. Depuis la fin de la décennie 2000 en particulier, on assiste à des changements rapides dans la manière d'interagir en ligne, caractéristiques du web 2.0. Celui-ci renvoie à un usage d'Internet qui va au-delà de la consultation, pour inclure de nouvelles façons de créer du contenu et de partager de l'information grâce au réseautage social ${ }^{59}$. Nicole B. Ellison et danah boyd définissent les sites de réseautage social ainsi :

A social network site is a networked communication platform in which participants 1) have uniquely identifiable profiles that consist of user-supplied content, content provided by other users, and/or system-provided data; 2) can publicly articulate connections that can

53 Christine Barré-de Miniac, Catherine Brissaud et Marielle Rispail, La littéracie : conceptions théoriques et pratiques d'enseignement de la lecture-écriture, coll Espaces discursifs, Paris, L'Harmattan, 2004. Les habiletés rattachées à ce concept peuvent varier en fonction du contexte social ou culturel.

54 «Technological Means to Access to Justice?» (15 septembre 2009) à la p 13, en ligne: Slaw $<$ http://www.slaw.ca/2009/09/15/technological-means-to-access-to-justice/>.

55 Maria Perez Crist, « The E-Brief : Legal Writing for an Online World» (2003) 33:1 N M Law Rev 49.

56 Dominique Cardon, La démocratie Internet : promesses et limites, coll République des idées, Paris, Seuil, 2010 à la p 8 [Cardon].

57 Communication Power, Oxford, Oxford University Press, 2009.

58 Cardon, supra note 56.

59 Millerand, Proulx et Rueff, supra note 11. 
be viewed and traversed by others; and 3) can consume, produce, and/or interact with streams of user-generated content provided by their connections on the site ${ }^{60}$.

Selon Alexandre Coutant et Thomas Stenger, les médias sociaux sont des sites Internet «1. dont le contenu est très largement produit par les internautes utilisateurs (principe UGC : user generated content), 2. qui regroupent des configurations sociotechniques très variées en termes de dynamique de participation (par intérêt, par amitié) et de visibilité (nature et finalité des données publiées en ligne) ${ }^{61}$. » Ces plateformes appelées «médias sociaux» regroupent donc des sites aux configurations et aux dynamiques de participation variées comme Facebook, Twitter, YouTube, Wikipédia et Google+ ${ }^{62}$. Certains auteurs utilisent le terme « web social » plutôt que « web 2.0 » pour souligner le fait que les bouleversements qu'il engendre ne sont pas seulement techniques, mais aussi sociaux, culturels et économiques ${ }^{63}$. Sur ces plateformes, les usagers évoluent dans des situations d'interactions où ils sont amenés à jouer un rôle en ligne important, notamment du point de vue de la production et de la circulation du contenu. Contrairement aux sites Internet traditionnels, ces plateformes, qui supposent une facilité de manipulation, impliquent la possibilité de consommer et de produire de l'information ${ }^{64}$ tout en modifiant et en relayant des contenus existants $^{65}$. Elles ont aussi pour particularité de favoriser des modalités de collaboration entre les internautes, et elles suggèrent une pluralité de pratiques et d'usages ${ }^{66}$. Le web social encourage ainsi « des formes inédites de partage du savoir ${ }^{67}$ qui rendent obsolète « le privilège d'accès à la publication dont bénéficiaient naguère les professionnels $\gg{ }^{68}$, dans une logique qui s'appuie sur la force du grand nombre ${ }^{69}$. C'est dans ce contexte que les sociologues des usages numériques se demandent dans quelle mesure ces espaces de discussion en ligne peuvent favoriser la construction collective de connaissance, mais aussi comment ils peuvent compléter ou aboutir à des formes de contestation des expertises traditionnelles en place $^{70}$.

Plusieurs auteurs s'intéressent manifestement aux transformations du domaine juridique en lien avec l'émergence des médias sociaux ${ }^{71}$. Une recherche dans les bases de données permet de constater que les

60 « Social Network Sites: Definition, History, and Scholarship » (2007) 13:1 J Comput-Mediat Commun 210-230 à la p 158.

61 « Médias sociaux : clarification et cartographie pour une approche sociotechnique » [2013] 70 Décisions Mark 107 à la p 115, DOI : 10.7193/dm.070.107.117.

62 Ibid

63 Millerand, Proulx et Rueff, supra note 11.

64 Webster, supra note 10 à la p 3.

65 Millerand, Proulx et Rueff, supra note 11.

66 Ibid

67 Cardon, supra note 56 à la $\mathrm{p} 8$.

68 Ibid à la p 10. Ce caractère inédit avait été anticipé avant l'arrivée d'Internet par le philosophe Walter Benjamin dans les années 1930 : «Pendant des siècles, un petit nombre d'écrivains se trouvaient confrontés à plusieurs milliers de lecteurs [mais] avec l'extension de la presse, qui n'a cessé de mettre à disposition du public de nouveaux organes [...], on vit un nombre croissant de lecteurs passer [...] du côté des écrivains. (Euvres III, Paris, Folio, 2000 à la p 95).

69 Millerand, Proulx et Rueff, supra note 11.

70 Florence Millerand, Lorna Heathon et David Myles, « Les reconfigurations sociales de l'expertise sur Internet » François Claveau et Julien Prud'homme, dir, Experts, sciences et sociétés, Montréal, Les Presses de l'Université de Montréal, 2018, 153.

71 À titre indicatif, une recherche dans la base de données HeinOnline effectuée le 6 mars 2018 (dans la section sur les périodiques juridiques) a permis de recenser 17308 résultats pour le terme « social media ». 
sujets suscitant le plus d'intérêt sont : l'administration de la preuve trouvée sur les médias sociaux 72 , les actes criminels commis sur les médias sociaux ${ }^{73}$, la liberté d'expression et la vie privée en ligne ${ }^{74}$ ainsi que la transformation de la pratique juridique par les médias sociaux ${ }^{75}$. On note également un certain intérêt pour les liens entre médias sociaux et information juridique ${ }^{76}$. À cet égard, plusieurs s'intéressent à l'utilisation des médias sociaux par les tribunaux dans un objectif de contrôle du discours, mais aussi de dissémination de l'information ${ }^{77}$. On retrouve des usages similaires par les organismes venant en aide aux personnes judiciarisées ${ }^{78}$. D'autres auteurs s'intéressent spécifiquement au média social Facebook, mais généralement par rapport à la mise en preuve des publications sur cette plateforme, ou à son utilisation par les professionnels du droit ${ }^{79}$. Les écrits portant sur les médias sociaux et l'autoreprésentation sont beaucoup plus rares ${ }^{80}$. Ils portent encore une fois sur l'utilisation des médias sociaux par les acteurs et institutions judiciaires pour diffuser de l'information, en particulier auprès des JNRA.

Certains auteurs soulignent plusieurs avantages potentiels liés aux médias sociaux pour les acteurs juridiques et les justiciables. Du point de vue des émetteurs de contenu, Suzanne Bouclin et Marie-Andrée Denis-Boileau, qui s'intéressent à l'itinérance, croient qu'il est possible de s'inspirer des organismes qui utilisent « les médias sociaux comme Facebook et Twitter comme moyens de joindre les personnes itinérantes et comme outils d'éducation juridique $»^{81}$. Ils permettraient donc de disséminer des ressources

72 Les termes « social media » et « evidence» sur HeinOnline ont donné à eux seuls 11225 résultats lors d'une recherche effectuée la même date. Voir notamment Agnieszka McPeak, "Avoiding Misrepresentation in Informal Social Media Discovery » (2014) 17 SMU Sci Tech Rev 581; Kristen L Mix, « Discovery of Social Media » (2011) 5 Fed Cts Rev 119; Paul W Grimm, Lisa Yurwit Bergstrom et Melissa M O’Toole-Loureiro, «Authentication of Social Media Evidence Symposium: Keynote Address » (2012) 36 Am J Trial Advocacy 433.

73 Notamment le harcèlement en ligne. Voir Peter T Elikann, «Criminal Law: Stalking through Social Media Case Comment » (2016) 98 Mass Law Rev 9; Daniel S Harawa, « Social Media Thoughtcrimes » (2014) 35 Pace Law Rev 366; Enrique A Monagas et Carlos E Monagas, «Prosecuting Threats in the Age of Social Media» (2015) 36 North Ill Univ Law Rev 57-78.

74 Roger Clarke, «Privacy and Social Media: An Analytical Framework» (2014) 23:169 J Inf Sc iv, DOI: 10.1016/j.bushor.2009.09.003; Stephen E Henderson, «Expectations of Privacy in Social Media » (2012) 31 Miss Coll Law Rev 227; Bethany N Whitfield, « Social Media @ Work : \#policyneeded Comment» (2013) 66 Ark Law Rev 843.

75 Raymond $\mathrm{H}$ Brescia et al, « Embracing disruption: how technological change in the delivery of legal services can improve access to justice » (2014) 78 Alb Rev 553; Andy Radhakant et Matthew Diskin, « How Social Media Are Transforming Litigation » (2013) 39 Litigation 17.

76 Supra note 16. Voir par ex Margaret D Hagan, « The User Experience of the Internet as a Legal Help Service: Defining standards for the next generation of user-friendly online legal services » (2016) 20 Va JL Tech 394.

77 Katherine Bladow et Joyce Raby, Using Social Media to Support Self-Represented Litigants and Increase Access to Justice, coll Future Trends in State Courts 2011, National Center for State Courts, 2011, en ligne : $<$ https://ncsc.contentdm.oclc.org/digital/collection/ctmedia/id/29/>; Pamela D Schutz et Andrew J Cannon, «Trial by Tweet? Findings on Facebook? Social Media Innovation or Degradation? The Future and Challenge of Change for Courts » (2013) 5:1 Int J Court Adm 25.

78 Bladow et Raby, supra note 79; Véronique Fortin, Taking the Law to the Streets: Legal and Spatial Tactics Deployed in Public Spaces to Control Protesters and the Homeless in Montreal, UC Irvine, 2015, en ligne: $<$ https://escholarship.org/uc/item/6hs4d5tp\#author>.

79 Une recherche dans la section juridique d'HeinOnline effectuée le 6 mars 2018 a permis de recenser 8129 résultats pour les mots-clés « social media » et « Facebook».

80 Toujours à titre indicatif, une recherche sur HeinOnline a recensé 272 résultats pour « social media » et « self-represented » à la même date.

81 Bouclin et Denis-Boileau, supra note 9 à la p 42. 
juridiques à des groupes et à des personnes qui n'y auraient pas accès autrement. Les médias sociaux permettent aussi une mise en dialogue entre les particuliers et les acteurs institutionnels ${ }^{82}$, comme les tribunaux et les organisations ${ }^{83}$. Du point de vue des récepteurs de contenu, ces outils peuvent être utilisés en combinaison ou au lieu des ressources institutionnelles, par exemple, pour évaluer au préalable quelles ressources consulter. Les médias sociaux pourraient donc permettre d'obtenir de l'information disponible ailleurs sur le web ${ }^{84}$, par exemple pour vérifier son admissibilité à l'aide juridique.

Or, les médias sociaux traduisent aussi des réalités émergentes dans le partage d'information dans la mesure où ils pourraient faciliter « l'autogestion juridique ${ }^{85}$. Dans ces contextes qui mettent à profit l'horizontalité dans les échanges, les frontières entre émetteurs et récepteurs de contenus deviennent poreuses. Contrairement à d'autres dynamiques de partage d'information, les justiciables ne sont pas seulement des récepteurs de contenus juridiques, mais jouent un rôle proactif dans leur production, leur circulation et dans l'adaptation du contenu préexistant à leur situation. En plus d'être faciles d'utilisation, les plateformes de production et d'exploitation de contenus comme Facebook permettent un accès instantané à un vaste réseau de personnes en une seule publication ${ }^{86}$. Celle-ci peut donc permettre de rejoindre des juristes, ou des personnes ayant vécu une expérience pertinente, comme un divorce. Ce processus serait difficile à reproduire en personne, car il nécessiterait un grand nombre de conversations avec des individus de différents cercles sociaux afin d'identifier ceux qui pourraient disposer d'informations ou d'expériences pertinentes.

Selon Cassandra Burke Robertson, les médias sociaux, en particulier Facebook, transformeront même la pratique juridique et démocratiseront l'information juridique. Ces transformations seront le fait de citoyens plutôt que de juristes ou d'institutions, dans la mesure où les premiers n'ont pas le choix de se tourner vers des ressources moins coûteuses que les services juridiques traditionnels. Ces changements dans la dissémination de l'information juridique s'opèreront donc par et pour les justiciables. Ils impliqueront surtout les JNRA de classe moyenne, qui ne peuvent accéder à des services juridiques, mais qui ont les ressources - notamment en termes de littératie - suffisantes pour être en mesure de répondre à leurs besoins en ligne. Cette approche «par le bas » plutôt que « par le haut» rejoint les sociologues de l'information qui démontrent que le dispositif sociotechnique du web social implique que les usagers jouent un rôle proactif et central dans la production de contenu ${ }^{87}$. Dans son ethnographie en ligne consacré à un forum sur le divorce, Carrie Paetcher observe que le web social permet même le développement d'une compréhension collective du processus juridique par les membres qui participent à la création de

82 Highfield, supra note 14.

83 Alysia Blackham et George Williams, « Australian courts and social media » (2013) 38:3 Altern Law J 170. Par exemple, au Québec, la Cour suprême est sur Facebook depuis 2011, mais ne publie du contenu que depuis décembre 2017 (« Cour suprême du Canada », en ligne : <https://www.facebook.com/coursupremeducanada >.) Certains tribunaux comme la Cour d’appel du Québec y sont aussi « représentés » par le biais de pages non officielles.

84 McGill, Bouclin et Salyzyn, supra note 27.

85 Ibid

86 Les plateformes interactives LegalZoom et LawPivot ont une visée d'information juridique pour le public : elles permettent à des avocats de répondre à des questions posées par des JNRA. Leurs réponses sont publiques pour en faire bénéficier les personnes ayant des besoins similaires.

87 Cardon, supra note 56; Manuel Castells, supra note 57. 
contenu $^{88}$. Tant le fait de poser une question que d'y répondre, ou de simplement commenter le contenu existant, contribue à ce processus collectif.

D'autres sites, comme les plateformes collaboratives de type wiki permettent le «crowdsourcing », un processus basé sur la collaboration d'individus pour résoudre des problèmes plutôt que d'obtenir l'assistance d'un spécialiste pour ce faire ${ }^{89}$. Elle permet donc à toute personne de participer à la production d'information sans la limiter à des experts reconnus ${ }^{90}$. Sa valeur se fonde sur le principe de sagesse des foules («wisdom of crowds 》) selon lequel les décisions collectives seraient plus susceptibles d'être bonnes lorsqu'elles sont prises par des personnes aux opinions diverses qui arrivent à des conclusions indépendantes ${ }^{91}$. Par opposition aux blogues, les wikis peuvent être corrigés et révisés par des contributeurs anonymes, d'où leur haut taux de fiabilité quant à l'information créée ${ }^{92}$. Selon Frank Webster, il s'agirait à tout le moins d'une solution de rechange viable au processus de validation de l'information par les experts ${ }^{93}$ qui se base non seulement sur les connaissances acquises dans un contexte professionnel, mais aussi sur les savoirs expérientiels des individus. Ce type de plateforme s'inscrit dans l'objectif de partage du savoir, un projet au cœur de la création d'Internet ${ }^{94}$ qui encourage la recherche collective et l'horizontalité dans le processus d'évaluation de la qualité des contributions ${ }^{95}$. Au Québec, le Wiki des arrêté-e-s est un bon exemple de ce type de plateforme : son objectif est le partage collaboratif d'information juridique pour les personnes arrêtées lors de manifestations ${ }^{96}$.

Ces mêmes auteurs évoquent aussi certaines limites des médias sociaux. Bouclin et Denis-Boileau croient qu'ils constituent « un bon moyen de tâter le terrain et d'obtenir de l'information de base, [mais qu'ils] ne sont toutefois pas suffisants pour outiller complètement un individu qui souhaite s'engager dans un processus judiciaire $»^{97}$. Paetcher observe aussi que les plateformes encouragent autant qu'elles entravent l'apprentissage de notions juridiques, notamment en raison des bulles informationnelles bridant les opinions minoritaires. À certaines occasions, le désir de partager de l'information se caractérise par la promotion presque «évangélique " (notre traduction) d'une solution plutôt qu'une autre : "One man posted on 19 separate occasions recommending [family mediation] to others, describing it in glowing terms $[\ldots] \gg^{98}$. Paetcher a aussi observé la présence de leaders d'opinion en ligne. La fréquence de leur activité conjuguée à la reconnaissance de leur statut d'expert par les autres internautes leur octroie une certaine autorité, sans pour autant garantir la pertinence de l'information partagée. On peut aussi penser

$88 \quad$ Paetcher, supra note 16.

89 Jeff Howe, «The Rise of Crowdsourcing», WIRED (juin 2006), en ligne: WIRED $<$ https://www.wired.com/2006/06/crowds/>.

90 Webster, supra note 10 à la p 246.

91 Friedrich A Hayek, «The Use of Knowledge in Society » (19450901) 35:4 Am Econ Rev 519; James Surowiecki, The Wisdom of Crowds, New York, Anchor, 2005 à la p 57.

92 Cass R Sunstein, Infotopia: How Many Minds Produce Knowledge, Oxford, Oxford University Press, 2006.

93 Webster, supra note 10.

94 Manuel Castells, The Internet Galaxy: Reflections on the Internet, Business, and Society, Oxford; New York, Oxford University Press, 2001. Internet étant d'ailleurs issu de la tradition de la « science ouverte » (Pierre Dardot et Christian Laval, Commun : Essai sur la révolution au XXI' siècle, Paris, La Découverte, 2014 à la p 162).

95 Philippe de Grosbois, Les batailles d'Internet. Assauts et résistances à l'ère du capitalisme numérique, Montréal, Écosociété, 2018.

96 «Wiki des arrêté-e-s », en ligne : <http://wikidesarretees.net//index.php?title=Accueil> (consulté le 12 novembre 2018).

97 Bouclin et Denis-Boileau, supra note 9 à la p 15.

98 Paetcher, supra note 16 à la p 400. 
qu'utiliser de l'information erronée ou décontextualisée est un risque qui peut avoir de lourdes conséquences pour les justiciables.

Le partage d'expériences individuelles en réponse à un questionnement juridique implique de replacer ces expériences dans leurs contextes spécifiques, qui peuvent être bien différents de la situation que vit l'internaute. Si ces limites peuvent se présenter dans d'autres contextes de recherche d'information que les médias sociaux, elles sont exacerbées par la dimension collaborative de ces plateformes. Or, au-delà des enjeux mis en lumière par les médias sociaux eux-mêmes, un autre défi concerne les chercheurs qui s'y intéressent: sur le plan empirique, comment appréhender les pratiques informationnelles des justiciables en ligne?

\section{COMPRENDRE LES PRATIQUES INFORMATIONNELLES DES JUSTICIABLES EN CONTEXTE NUMÉRIQUE}

Nous observons que les chercheurs en droit documentent rigoureusement l'implantation des TIC dans le système judiciaire, ainsi que le cadre juridique de cette implantation. Les articles portant sur le droit et les médias sociaux s'intéressent surtout à la transformation de la pratique des professionnels du droit et à la mise en preuve des contenus qui s'y trouvent. De rares articles s'intéressent aux avantages et aux limites de la présence de l'information juridique sur les médias sociaux, que ce soit pour les organisations ou les justiciables. Toutefois, les pratiques informationnelles des justiciables dans les médias sociaux et le rôle qu'elles jouent sur le plan de l'accès à la justice restent à documenter. Davantage de recherche est également nécessaire pour comprendre dans quelle mesure ces avantages et ces limites se déploient sur le terrain. Si nous avons mis en lumière plusieurs particularités des médias sociaux à cet égard, il importe de savoir dans quelle mesure on y retrouve effectivement ces pratiques et dans quels différents contextes d'échange elles émergent.

\section{A. L'accès à la justice à l'aune de la sociologie du numérique}

Bien qu'ils ne se soient pas spécifiquement intéressés à l'information juridique, dans les dernières années, les théoriciens de la communication et de l'information ont transposé leur intérêt depuis les effets des technologies sur la société vers ce que les individus peuvent faire avec les technologies ${ }^{99}$. Ils rompent ainsi avec un déterminisme technique pour lequel la technologie est une force extérieure qui détermine le social $^{100}$, mais aussi avec la posture du déterminisme social, qui considère le développement des

99 Webster, supra note 10. On retrouve cependant un foisonnement de publications sur les usages d'Internet en matière de santé. Voir notamment Yan Zhang, Dan He et Yoonmo Sang, «Facebook as a Platform for Health Information and Communication: A Case Study of a Diabetes Group » (2013) 37:3 J Med Syst, DOI : 10.1007/s10916-013-9942-7; William B Lober et Janine L Flowers, « Consumer empowerment in health care amid the internet and social media » (2011) 27:3 Semin Oncol Nurs 169, DOI : 10.1016/j.soncn.2011.04.002; Madeleine Akrich et Cécile Méadel, «Internet : intrus ou médiateur dans la relation patient/médecin?» (2009) 8:2 Santé Société Solidar 87-92, DOI : 10.3406/oss.2009.1362; Christine Seux, «Les disparités sociales des usages d'internet en santé : Effets combinés des socialisations familiales et des sources informationnelles» (2018) 208:2 Réseaux 63, DOI : 10.3917/res.208.0063; Dominique Michaud et al., «Le Web 2.0 pour soutenir le réseautage en santé mentale au Québec » (2015) 40:1 Santé mentale au Québec 227, DOI : 10.7202/1032392ar.

100 Francis Jauréguiberry et Serge Proulx, Usages et enjeux des technologies de communication, Toulouse, Érès, 2011 à la p 10, en ligne : <https://hal.archives-ouvertes.fr/hal-01427512>. 
innovations techniques «comme un mécanisme de reproduction des inégalités, des hiérarchies et des fractures du tissu social ${ }^{101}$. Il s'agit d'adopter une approche axée sur la mise en usage des dispositifs sociotechniques, qui se fonde sur une posture épistémologique constructiviste, en rupture à la fois avec le déterminisme social et le déterminisme technologique. Étudier les usages d'une technologie consiste donc à observer, à décrire et à expliquer ce que les individus font effectivement avec les objets, outils et dispositifs techniques ${ }^{102}$. Certes, les technologies ne sont pas neutres : pour le juriste Lawrence Lessig, l'architecture d'Internet est une forme de loi qui détermine ce que les gens peuvent ou ne peuvent pas y faire ${ }^{103}$. Les plateformes numériques comme Facebook participent au cadrage des interactions dans la mesure où elles possèdent des affordances spécifiques qui suggèrent certaines utilisations plutôt que d'autres ${ }^{104}$. Or, cette architecture est considérablement modalisée par l'intervention humaine, qu'elle soit intentionnelle ou accidentelle. Les technologies peuvent même être utilisées à d'autres fins que celles pour lesquelles elles ont été conçues à l'origine ${ }^{105}$. Le design, les significations et les effets d'une technologie changent constamment au fur et à mesure que ses usagers la transforment et que son environnement évolue ${ }^{106}$. En somme, les approches issues de la sociologie du numérique mettent en exergue le fait que les TIC sont des constructions humaines à la fois déterminantes et déterminées, et que leur rapport avec la société est dialectique. Elles permettent également d'échapper à une posture techno utopiste ou techno sceptique ${ }^{107}$ dans le regard que l'on porte sur les liens entre droit et technologies. Comme l'explique Dominique Boullier, ces approches ne se cantonnent pas à un domaine spécifique, puisque « le numérique a ceci de particulier qu'il est "pervasif", c'est-à-dire qu'il pénètre toutes nos activités, des plus intimes aux plus collectives $»^{108}$. À l'ère où les phénomènes juridiques comportent une importante dimension numérique et peuvent s'exprimer à travers le numérique, nous croyons que la recherche en droit doit s'inspirer de cette contribution.

Sur le plan méthodologique, ce « déplacement de l'intérêt centré sur la technologie vers les usagers » implique un recours aux méthodologies de type ethnographiques et microsociologiques selon une approche constructiviste ${ }^{109}$. Les méthodes de recherche en ligne en particulier, notamment l'ethnographie en ligne, prennent acte de l'emprise croissante de la communication médiatisée par le numérique dans nos activités quotidiennes. Elles permettent de centrer notre regard sur l'expérience des internautes afin de mieux comprendre leurs pratiques, les significations qu'ils attribuent à ces pratiques et leurs besoins. La démocratisation de l'accès à Internet requiert en effet de développer des méthodes pour « appréhender

101 Florence Millerand, « Usages des NTIC : les approches de la diffusion, de l'innovation et de l'appropriation (1 ${ }^{\text {ère }}$ partie) » (1998) 2:1 COMMposite 1 à la p 4.

102 Jauréguiberry et Proulx, supra note 100 à la p 24.

103 Code and Other Laws of Cyberspace, Basic Books, 1999.

104 Serge Proulx, supra note 46. Le terme «affordance» renvoie à la capacité d'un objet à suggérer sa propre utilisation (Donald A Norman, « Affordance, Conventions, and Design » (1999) 6:3 Interactions 38, DOI : 10.1145/301153.301168.)

105 George Basalla, The Evolution of Technology, Cambridge, Cambridge University Press, 1988 à la p 7.

106 Sur la question de comment les usagers structurent les technologies, voir Nelly Oudshoorn et Trevor Pinch, dir, How Users Matter: The Co-Construction of Users and Technology. Cambridge, MA, MIT Press, 2005.

107 Andrew Feenberg, Pour une théorie critique de la technique, Montréal, Lux, 2014.

108 Sociologie du numérique, Paris, Armand Colin, 2016 à la p 6.

109 Florence Millerand, «Usages des NTIC : les approches de la diffusion, de l'innovation et de l'appropriation ( $2^{\mathrm{e}}$ partie) » (1998) 3:1 COMMposite 54 à la p 8. 
[s]es impacts sociaux et culturels $»^{110}$ dans une démarche compréhensive héritée des sciences sociales. Il s'agit de réfléchir à des stratégies pour adapter les pratiques ethnographiques au contexte numérique contemporain plutôt que de concevoir l'ethnographie "virtuelle» comme une catégorie distincte de l'ethnographie ${ }^{111}$. De telles approches peuvent permettre de garder une trace des interactions entre les internautes et des contenus échangés dans le contexte de leurs activités quotidiennes, par contraste avec des enquêtes basées sur leurs déclarations ou encore des situations de test. Dans la mesure où nous nous intéressons aux problématiques d'accès à la justice, il s'agit ici plus précisément de comprendre l'influence d'Internet sur la capacité d'agir des justiciables.

Finalement, en plus de ces considérations théoriques et méthodologiques, le contexte socioéconomique de l'accès à la justice évoqué précédemment peut aussi justifier le recours aux méthodes de recherche en ligne. Documenter les problèmes d'accès à la justice dans toute leur complexité implique de ne pas se limiter à observer les activités dans les tribunaux ${ }^{112}$. Hormis en matière familiale, les usagers des tribunaux civils sont majoritairement des entreprises ou des institutions gouvernementales et non gouvernementales ${ }^{113}$. Une étude démontre que seulement $10 \%$ de la population québécoise ont les moyens d'aller devant les tribunaux, en plus des $10 \%$ qui sont admissibles à l'aide juridique ${ }^{114}$. Bien que ces données ne soient pas récentes, le problème de l'inaccessibilité aux services juridiques reste actuel et majeur. Lafond précise :

Si on compare les coûts d'un procès avec le revenu moyen des Québécois, on reste étonné de constater à combien de semaines de salaire ils correspondent. Pour certaines personnes - les personnes seules ou avec un revenu moyen -, cela peut facilement représenter l'équivalent de deux ans de salaire ${ }^{115}$.

Même sans les honoraires d'avocat, les personnes non représentées doivent débourser des montants substantiels (frais judiciaires, de signification des procédures, d'assignation des témoins, d'expertise, etc.). En définitive, on peut penser que les JNRA ne représentent qu'une partie des personnes qui ont des problèmes juridiques, mais qui n'ont pas les moyens de les régler. Or, d'autres justiciables sont invisibles depuis les tribunaux ou même d'autres instances de résolution de conflits. Comme l'affirme Lafond: « [u]ne conception de l'accès à la justice réaliste et concrète doit tenir compte des situations absentes de l'enceinte judiciaire $\rangle^{116}$ (nos soulignés). C'est sans compter les autres barrières, notamment informationnelles et psychologiques, qui peuvent freiner le recours à des démarches formelles. Nous pensons donc que les médias sociaux, de plus en plus ancrés dans le quotidien, peuvent être un observatoire de rapports inégalitaires dans l'accès au système de justice et à l'information juridique. Il s'agit donc, en plus de documenter l'impact des médias sociaux sur la capacité d'agir, de comprendre la capacité d'agir des individus par le biais des médias sociaux.

110 Madeleine Pastinelli, « Pour en finir avec l'ethnographie du virtuel! : Des enjeux méthodologiques de l'enquête de terrain en ligne» (2011) 35:1-2 Anthropologie et Sociétés 35 à la p 105.

111 Christine Hine, Ethnography for the Internet: Embedded, Embodied and Everyday, Londres, Bloomsbury Academic, 2015.

112 Lafond, supra note 1.

113 Ibid à la p 43.

114 Yan Muckle, «Y a-t-il encore une justice? » (juillet 2000), L'actualité, Montréal, à la p 18.

115 Supra note 3 à la p 53.

116 Lafond, supra note 1 à la p 18. 
Les terrains en contexte numérique ne sont pas les seuls qui permettent de documenter la réalité des personnes ayant des besoins juridiques en dehors des tribunaux. Une étude québécoise s'est intéressée aux services de première ligne qui leur sont destinés et notamment au cas d'une clinique juridique communautaire ${ }^{117}$. Or, les participantes à l'étude ont soutenu qu'un des défis importants rencontrés par la clinique était le fait que la population ignorait l'existence même de ces services. Cette étude révèle aussi que les justiciables qui avaient eu recours à de l'information juridique en ligne estimaient que celle-ci était bien souvent source de confusion en raison de la complexité du langage employé, des mots-clés à connaître pour faire des recherches, de la difficulté à appliquer l'information juridique à leur propre situation ainsi que des contradictions entre certaines informations. Ces résultats permettent de se demander quel rôle jouent les médias sociaux dans le processus de recherche d'information des justiciables. Mais elles démontrent aussi que les activités en ligne et hors-ligne ne doivent pas être observées en silo et que les unes peuvent permettre de mieux comprendre les autres.

Les terrains de recherche sur les médias sociaux constituent donc plus qu'une approche pour documenter les pratiques liées à leur utilisation. Ils constituent un moyen privilégié pour entrer en contact avec des justiciables qui ne sont pas devant les tribunaux ou d'autres instances de résolution de conflits et qui n'y seront peut-être jamais, mais qui sont aux prises avec des problématiques juridiques bien réelles. Voyons maintenant un aperçu de ce type de terrain de recherche.

\section{B. Typologie des pages et groupes Facebook québécois contenant de l'information juridique}

À la lumière des approches de la sociologie du numérique, nous proposons maintenant un survol des pages et groupes qui contiennent de l'information juridique en contexte québécois sur le réseau social Facebook ${ }^{118}$. Ce choix s'explique d'abord par le fait qu'il s'agit de la principale plateforme de réseautage social en ligne avec plus de 1,5 milliard d'utilisateurs « actifs » ${ }^{119}$. Au Québec, il s'agit du principal réseau social utilisé pour accéder à de l'information en $2016^{120}$. Les usages de ce média sont « désormais si immédiats, quotidiens et communs que le service s'est banalisé au point de devenir une pratique sociale très ordinaire $\rangle^{121}$ et s'intègrent ainsi dans une pluralité d'activités comme la recherche d'information. En outre, contrairement à d'autres plateformes comme les wikis, qui ont généralement une finalité et un public très restreints, Facebook rassemble en un même espace plusieurs types de contenus, qu'il s'agisse de témoignages personnels des internautes ou du partage d'hyperliens provenant de sites externes qui peuvent

117 «La force du suivi personnalisé pour les personnes autoreprésentées », supra note 52.

118 Les pages ont été conceptualisées pour permettre à des figures publiques, à des organisations et à d'autres entités de créer une présence publique et authentique sur Facebook. Elles sont visibles pour tous les membres du média social. Le réseau sert davantage d'espace de communication pour les petits groupes qui ont des intérêts communs. À la manière d'un forum de discussion, les groupes permettent aux internautes de se rassembler autour d'une cause, d'une activité ou d'un enjeu partagé. Les échanges y sont généralement plus horizontaux que sur une page, où les discussions sont axées autour du contenu partagé par le gestionnaire de la page. (Matt Hicks, « Facebook Tips: What's the Difference between a Facebook Page and Group? » (février 2010), en ligne : Facebook <https://www.facebook.com/notes/facebook/facebook-tips-whatsthe-difference-between-a-facebook-page-and-group/324706977130/> [consulté le 16 novembre 2018]).

119 Irène Bastard et al, «Facebook, pour quoi faire ? : Configurations d'activités et structures relationnelles » (2017) 8:1 Sociologie 57 à la p 58.

120 Internet pour s'informer et communiquer, Volume 7-Numéro 7, coll NETendances 2016, CEFRIO, 2017, en ligne : $<\mathrm{https}$ ///cefrio.qc.ca/fr/enquetes-et-donnees/netendances2016-internet-pour-sinformer-et-communiquer/> (consulté le 14 décembre 2018).

121 Bastard et al, supra note 118 à la p 58. 
être rattachés à des organisations ou des bureaux juridiques. Ce média est ainsi particulièrement pertinent pour notre recherche, puisqu'il s'insère dans un système hybride qui rassemble à la fois des contributions citoyennes et des contributions des plateformes traditionnelles ${ }^{122}$. Il peut dès lors être intéressant de comprendre comment ces deux types de contributions s'articulent dans le discours des internautes.

Cette démarche s'inscrit dans une recherche de type ethnographique en cours sur cette plateforme. Lors de la première phase de recherche, entre les mois de décembre 2017 et de mars 2018, nous avons mené une veille d'observation de différentes pages et différents groupes qui mentionnaient un lien explicite avec l'information ou avec les institutions juridiques ${ }^{123}$. En plus de la recherche par mots-clés, nous avons sélectionné nos groupes par le biais d'autres procédés : les suggestions de l'algorithme Facebook au fur et à mesure du processus de veille, ainsi que la technique «boule de neige ${ }^{124}$ consistant ici à mobiliser notre réseau personnel. Nous avons recensé l'ensemble des groupes et pages pertinentes et les avons classés selon différents critères : taille du groupe, niveau de confidentialité, domaines de droit abordés, présence ou non d'administrateur, types de contenus présentés et fréquence des échanges en lien avec le droit par rapport à d'autres types de contenu ${ }^{125}$. À la suite d'une analyse comparative de ces différents espaces, nous avons élaboré, de manière inductive, une typologie des pages et groupes Facebook contenant de l'information juridique. Considérant le thème de notre recherche, à savoir les pratiques informationnelles par et pour les internautes, où ils sont émetteurs et récepteurs de contenus, nous avons consulté, mais exclu les pages destinées spécifiquement aux professionnels du droit. Afin de préserver la confidentialité des internautes, nous avons altéré les noms exacts des groupes fermés comptant peu de membres (moins de 300) ${ }^{126}$. Précisons qu'en raison de l'abondance des pages, groupes et contenus disponibles sur Facebook de manière générale, cette recension n'est pas exhaustive et vise avant tout à présenter des idéaux types de pratiques qui peuvent s'y retrouver ${ }^{127}$. Nous avons recensé l'ensemble des groupes et pages pertinentes et les avons classés selon différents critères.

122 Dans le contexte des médias d'information, Andrew Chadwick est à l'origine du concept de «système médiatique hybride » pour désigner l'interaction entre les nouveaux médias " citoyens » et les médias traditionnels, qui structurent ensemble la couverture de l'actualité, l'un interagissant avec l'autre. Ce système permet aux médias, aux acteurs politiques, mais aussi au public d'avoir une influence sur la logique médiatique, sans que le pouvoir ne soit réservé aux deux premiers. Il serait ainsi intéressant d'analyser dans quelle mesure cette théorie s'applique au-delà des contenus journalistiques, notamment quant à l'information juridique (The Hybrid Media System: Politics and Power, Oxford ; New York, Oxford University Press, 2013.)

123 Nous avons amorcé la recherche sur le moteur de recherche de Facebook avec les mots-clés «droit», " information juridique » et « conseil juridique». Nous avons par la suite élargi notre recherche aux suggestions de pages et de groupes affichés qui avaient été trouvées et aux mots-clés qui revenaient fréquemment dans ces premiers résultats, par exemple le terme « aide juridique » ou « assistance juridique». Les messages publiés à partir de comptes personnels des membres du média social ne sont pas pris en compte dans cette recension de contenu.

124 Lorraine Savoie-Zajc, « Comment peut-on construire un échantillonnage scientifiquement valide? » [2007] 5 Rech Qual 99.

125 Le détail des pages analysées est présenté sous forme de tableau en annexe.

126 Les contenus d'un groupe « public » (ou « ouvert») sont accessibles à tous les membres de Facebook. Par contre, même si le groupe est public, les internautes doivent en devenir membres pour partager des contenus et réagir aux contenus existants. Dans le cas où le groupe est dit «fermé », ses contenus (discussions, photos) ne seront visibles que par les membres du groupe.

127 Un idéal type est une catégorie, ou une représentation modélisée, qui permet de comprendre certains phénomènes ou de tenter de les théoriser, sans pour autant prétendre que les caractéristiques de ces types se retrouvent toujours et parfaitement dans les phénomènes observés. On obtient l'idéal type lorsqu'on accentue délibérément les traits les plus significatifs selon 
Si nous avons volontairement restreint notre recherche aux groupes en lien avec le droit québécois, soulignons que la création de ces espaces a une dimension internationale. Par exemple, aux États-Unis, le groupe Legal Advice (Free) rassemble environ 227000 internautes qui posent des questions juridiques sur une base quotidienne dans plusieurs domaines de droit ${ }^{128}$. Il y a un fort niveau d'interaction et les réponses y sont parfois très longues et détaillées : on semble y retrouver une certaine dynamique d'entraide. On retrouve aussi plusieurs groupes d'«aide juridique» en droit français qu'ils soient généraux ou spécialisés, en droit du travail par exemple.

La première catégorisation vise les pages et groupes dont le contenu est destiné en tout ou en partie à l'information juridique. Sur ces espaces, on retrouve une quantité importante de publications d'internautes qui formulent des questions par rapport à leur situation juridique, ainsi que les réponses d'autres internautes. Cette catégorisation regroupe les pages associées à des organismes juridiques qui publient des articles d'information, comme Éducaloi. Les internautes y posent généralement leurs questions sous forme de commentaire sous les publications principales, en demandant des précisions par rapport à leur situation personnelle. La question est généralement en lien avec un article publié par l'organisme, mais peut porter sur un autre domaine ou une autre question de droit connexe. D'autres internautes vont fréquemment répondre eux-mêmes à ces questions ou demander des précisions ${ }^{129}$. Il arrive aussi que le gestionnaire de la page réagisse à ces demandes en renvoyant à un article d'information de son propre organisme ou d'une autre institution, comme celle d'un tribunal ou d'un service gouvernemental. Cette catégorisation vise aussi plusieurs groupes et des pages gérées par des internautes-administrateurs qui ne sont donc pas des pages officiellement rattachées à une organisation ${ }^{130}$. On y retrouve plusieurs publications d'internautes formulant des besoins d'information ou de support juridique. Une internaute peut par exemple faire état de sa situation personnelle, demander si des recours sont envisageables et comment elle pourrait intenter une démarche judiciaire le cas échéant. Sur ce dernier point, il peut s'agir par exemple de demander spécifiquement quelles informations intégrer dans une mise en demeure. D'autres internautes fournissent des réponses sous forme de commentaires sous cette publication. Le niveau de précision et de directivité de ces réponses est variable, et ces internautes font souvent état de leur propre expérience avec une démarche judiciaire en guise de réponse. À d'autres occasions, les internautes vont relayer du contenu existant en plus ou plutôt que de formuler eux-mêmes une réponse, en donnant par exemple un hyperlien vers un article d'information juridique sur Internet.

La deuxième catégorisation vise les groupes rassemblant des internautes qui vivent ou ont vécu une situation commune dans le système de justice, comme une arrestation de masse à la suite d'une manifestation ou un recours collectif. Dans le contexte d'une manifestation, par exemple, les échanges entre les membres contiennent de l'information générale sur les domaines de droit applicables (droit criminel et pénal), mais aussi sur la jurisprudence en lien avec l'infraction reprochée, les faits matériels importants lors de l'arrestation et l'évolution de la démarche judiciaire. L'objectif de ces groupes implique que l'information partagée ait une finalité très précise, qui pourrait être trop spécifique pour des personnes

la perspective adoptée. Il sert donc à construire un modèle de phénomène social et permet de refléter une perspective liée au but de ce modèle : Max Weber, Essais sur la théorie de la science, traduit par Julien Freund, Paris, Librairie Plon, 1965.

30 Impliquant ainsi un détournement des usages encouragés par les concepteurs de Facebook à l'origine, à savoir de réserver les pages à des entités officiellement reconnues. 
qui ont un problème similaire, mais qui ne font pas partie de la démarche en question. On constate donc que ces groupes sont utilisés par un bassin restreint de personnes qui est prédéterminé par un évènement s'étant matérialisé hors-ligne. Le nombre de membres est d'ailleurs souvent peu élevé (moins de 300 personnes). Si les groupes recensés concernent essentiellement des arrestations de masse, on peut penser que d'autres groupes aux usages similaires existent pour d'autres démarches et en particulier pour des recours collectifs ${ }^{131}$.

La troisième catégorisation de page Facebook vise les groupes dont l'objectif, selon leur description, n'est pas de partager de l'information juridique, mais où l'on y formule des demandes d'information ou de support juridique de manière ponctuelle. Les thèmes de ces groupes sont très variés (logement, sport, immigration), mais on peut penser que le très grand nombre de membres du groupe (généralement plus de 10000 membres et parfois plus de 40 000) fait en sorte que les internautes mettent à profit la force du nombre pour trouver une réponse à leur question. Ce type d'usages fait écho aux écrits sur les affordances des médias sociaux, qui permettent de rejoindre un très grand nombre de personnes, dont certaines ayant une expérience pertinente, en une seule publication. Mentionnons finalement qu'en raison de leur portée très générale, les groupes identifiés pendant notre veille ne sont probablement qu'une partie de ceux qui entrent dans cette catégorie.

Tableau 1. Typologie des pages Facebook contenant de l'information juridique

\begin{tabular}{|l|l|l|l|}
\hline Catégorie de page & Gestionnaires de & $\begin{array}{l}\text { Nombre } \\
\text { membres }\end{array}$ & Niveau d'interaction \\
\hline $\begin{array}{l}\text { Pages et groupes } \\
\text { destinés au droit (en } \\
\text { tout ou en partie) }\end{array}$ & $\begin{array}{l}\text { Organismes publics ou } \\
\text { communautaires/regroupements } \\
\text { d'intérêt en fonction de } \\
\text { situations spécifiques }\end{array}$ & $\begin{array}{l}\text { Variable } \\
\text { les grable. Souvent élevé pour } \\
\text { (plusieurs contributions par } \\
\text { jour et plusieurs réponses aux } \\
\text { questions formulées) }\end{array}$ \\
\hline $\begin{array}{l}\text { Pages et groupes } \\
\text { destinés au droit } \\
\text { dans le cadre d'un } \\
\text { évènement précis }\end{array}$ & $\begin{array}{l}\text { Groupes restreints en fonction } \\
\text { de la participation à un } \\
\text { évènement }\end{array}$ & Faible & $\begin{array}{l}\text { Variable en fonction de } \\
\text { l'évènement (pas ou peu } \\
\text { d'interactions après la fin } \\
\text { d'une étape importante } \\
\text { relative à l'évènement) }\end{array}$ \\
\hline $\begin{array}{l}\text { Pages qui ne sont pas } \\
\text { consacrées au droit }\end{array}$ & $\begin{array}{l}\text { Regroupement en fonction de } \\
\text { thématiques d'intérêt }\end{array}$ & $\begin{array}{l}\text { Très élevé, mais les } \\
\text { interactions relatives au droit } \\
\text { demeurent ponctuelles }\end{array}$ \\
\hline
\end{tabular}

On constate à la lumière de cette typologie que les pratiques informationnelles en matière juridique varient énormément dans les médias sociaux : elles peuvent viser des groupes aux objectifs très différents, avec un nombre de membres et des niveaux d'interaction très variés. Leurs contenus peuvent ou non être accessibles à tout membre du réseau social. Une partie de ces contenus se retrouve dans des groupes qui n'ont aucun lien apparent avec le droit ou la justice selon leur description. Il serait d'ailleurs impossible de recenser l'ensemble des questions juridiques formulées sur un média social comme Facebook. En

131 Les travaux de la professeure Catherine Piché révèlent d'ailleurs un meilleur taux d'indemnisation pour les membres des recours collectifs lorsque les avis d'indemnisation sont diffusés sur Internet et sur les médias sociaux : Catherine Piché, « User des technologies et des médias sociaux pour mieux communiquer avec les membres et pour mieux indemniser dans l'action collective », Colloque Clarity 2018, Montréal, 26 octobre 2018. 
raison du niveau d'interaction relatif aux questions de nature juridique qu'on y retrouve, nous avons choisi de nous intéresser à des groupes de la première catégorie aux fins de notre recherche ethnographique.

\section{CONCLUSION}

Dans le présent article, nous avons démontré que les échanges en lien avec l'information juridique sont bel et bien présents sur les médias sociaux. Ces pratiques évoluent dans un contexte où il existe plusieurs barrières socioéconomiques et informationnelles en matière d'accès à la justice. Elles coévoluent aussi avec l'intégration des TIC dans le monde juridique pour pallier les problèmes d'accès à la justice. Or, par opposition à d'autres types de plateformes numériques, les médias sociaux ont pour particularité de mettre en scène des internautes qui sont à la fois émetteurs et récepteurs de contenus d'information juridique et qui contribuent collectivement à structurer la nature et la circulation de cette information. Ces internautes contribuent d'ailleurs à relayer les contenus déjà existants de plateformes traditionnelles comme les sites d'information gouvernementaux. Les pratiques informationnelles des internautes sur ces plateformes se déploient dans différents espaces et contextes précis, qu'ils soient spécifiquement liés à la recherche d'information juridique, à un évènement hors-ligne qui rassemble une communauté de justiciables, ou encore à une communauté liée par des activités comme la recherche de logement ou la pratique d'un sport. Les plateformes d'information juridique sur les médias sociaux et leurs usages devront faire l'objet de recherches empiriques afin de comprendre le rôle qu'elles peuvent jouer sur le plan de l'accès à la justice et notamment sur la capacité d'agir individuelle et collective des internautes. Notre analyse permet de déterminer qu'elles devront être appréhendées au moyen d'une approche microsociologique ou ethnographique afin de comprendre leurs implications pour les justiciables. Or, une telle approche doit tenir compte de la situation socioéconomique plus large dans laquelle ces pratiques informationnelles se déploient, à savoir les problèmes d'accès à la justice évoqués dans le présent article. La recherche en droit doit en effet s'attarder à comprendre ce que ces pratiques révèlent sur l'accès à la justice et la constellation de problèmes auxquels les justiciables font face.

Un croisement entre les champs d'études du droit et de la sociologie du numérique, théorique comme méthodologique, devient de plus en plus nécessaire pour documenter la réalité contemporaine des justiciables. À cet égard, les méthodes de recherche en ligne pourraient non seulement permettre de comprendre un phénomène numérique et sociojuridique dans les angles morts de la recherche actuelle, mais aussi de rejoindre des internautes qui ont des problèmes juridiques bien réels, mais qui n'ont pas accès au système de justice en raison des barrières financières et informationnelles qu'il pose.

Les possibilités de ces approches théoriques et méthodologiques pour les études sur les usages numériques ne se limitent pas aux médias sociaux. Ces approches pourraient notamment être utilisées dans le champ de la cyberjustice pour comprendre les appropriations des nouvelles technologies judiciaires par les justiciables (vidéoconférence, plateformes de règlements des différends en ligne) et leurs impacts sur les trajectoires judiciaires. S'intégrant aux approches en droit et société et en études culturelles du droit, elles pourraient aider à mieux comprendre les représentations du droit et de la justice de manière générale. Finalement, ces stratégies de recherche qualitatives pourraient débroussailler d'importantes pistes de recherche pour la production d'études statistiques sur les profils des justiciables susceptibles d'utiliser les TIC pour répondre à leurs besoins juridiques. Par le biais des méthodes 
« natives » des médiums numériques ${ }^{132}$, elles pourraient aussi précéder des stratégies d'extraction de données massives («big data ») afin de visualiser où et comment circule l'information issue des sites Internet d'information juridique et de jurisprudence. En somme, ces approches pourraient permettre de mieux cibler à qui profitent ces nouvelles technologies et à quelles fins. Au premier chef, ce dialogue entre droit et communication pourrait permettre de mieux cartographier les problèmes d'accès à la justice à l'ère du numérique.

\section{$\underline{\text { Annexe }}$}

1. Les pages et groupes consacrés en tout ou en partie au droit

\begin{tabular}{|c|c|c|c|c|c|c|c|c|}
\hline & Type & $\begin{array}{c}\text { Ouvert } \\
\text { ure }\end{array}$ & $\begin{array}{c}\text { Nombre } \\
\text { approximatif } \\
\text { d'internautes }\end{array}$ & $\begin{array}{l}\text { Description des } \\
\text { objectifs de la } \\
\text { page }\end{array}$ & $\begin{array}{l}\text { Domaines de } \\
\text { droits abordés }\end{array}$ & $\begin{array}{l}\text { Présence de } \\
\text { modération }\end{array}$ & $\begin{array}{l}\text { Activité } \\
\text { récente* }\end{array}$ & $\begin{array}{l}\text { Types de } \\
\text { contenus } \\
\text { présents }\end{array}$ \\
\hline $\begin{array}{l}\text { Assez } \\
\text { du } \\
\text { DPJ }\end{array}$ & $\begin{array}{l}\text { Group } \\
\mathrm{e}\end{array}$ & $\begin{array}{l}\text { Public* } \\
*\end{array}$ & $\begin{array}{l}\text { Plus de } \\
13000\end{array}$ & $\begin{array}{l}\text { Partager son } \\
\text { expérience avec } \\
\text { le Directeur de } \\
\text { la protection de } \\
\text { la jeunesse }\end{array}$ & $\begin{array}{l}\text { Droit de la } \\
\text { protection de la } \\
\text { jeunesse }\end{array}$ & $\begin{array}{l}8 \\
\text { administrateurs } \\
\text { et modérateurs, } \\
\text { certains } \\
\text { intervenants } \\
\text { interviennent } \\
\text { fréquemment } \\
\text { pour fournir de } \\
\text { l'information } \\
\text { juridique }\end{array}$ & Oui & $\begin{array}{l}\text { Questions à } \\
\text { savoir comment } \\
\text { déposer une } \\
\text { plainte ou } \\
\text { amorcer une } \\
\text { démarche } \\
\text { judiciaire, récits } \\
\text { d'expérience } \\
\text { abordant des } \\
\text { problèmes } \\
\text { juridiques, } \\
\text { questions par } \\
\text { rapport au } \\
\text { processus } \\
\text { judiciaire. }\end{array}$ \\
\hline $\begin{array}{l}\text { Cour } \\
\text { suprê } \\
\text { me du } \\
\text { Canad } \\
\text { a }\end{array}$ & $\begin{array}{l}\text { Page } \\
\text { officie } \\
\text { lle*** }^{* *}\end{array}$ & $\begin{array}{l}\text { Publiqu } \\
\mathrm{e}\end{array}$ & Une centaine & $\begin{array}{l}\text { Non mentionné, } \\
\text { mention de } \\
\text { l'historique de la } \\
\text { cour }\end{array}$ & Général & $\begin{array}{l}\text { Par les } \\
\text { gestionnaires de } \\
\text { la page }\end{array}$ & Oui & $\begin{array}{l}\text { Photos } \\
\text { d'évènements. } \\
\text { Des résumés en } \\
\text { langage clair y } \\
\text { seront } \\
\text { disponibles } \\
\text { prochainement. } \\
\text { On précise ne } \\
\text { pas répondre } \\
\text { aux demandes } \\
\text { d'information } \\
\text { juridique : lien } \\
\text { vers des } \\
\text { références sur le } \\
\text { site de } \\
\text { l'institution } \\
\text { pour ce type de } \\
\text { demandes. }\end{array}$ \\
\hline $\begin{array}{l}\text { DPJ } \\
\text { Vos } \\
\text { Questi } \\
\text { ons } \\
\text { Anony }\end{array}$ & Page & $\begin{array}{l}\text { Publiqu } \\
\text { e }\end{array}$ & Environ 150 & $\begin{array}{l}\text { Publication de } \\
\text { questions de } \\
\text { manière } \\
\text { anonyme sur le } \\
\text { DPJ }\end{array}$ & $\begin{array}{l}\text { Droit de la } \\
\text { protection de la } \\
\text { jeunesse }\end{array}$ & $\begin{array}{l}\text { Par le } \\
\text { gestionnaire de } \\
\text { la page }\end{array}$ & Non & $\begin{array}{l}\text { Quelques } \\
\text { questions } \\
\text { juridiques à } \\
\text { savoir comment } \\
\text { faire valoir ses }\end{array}$ \\
\hline
\end{tabular}

132 Richard Rogers, Digital Methods, Cambridge, MIT Press, 2013. 


\begin{tabular}{|c|c|c|c|c|c|c|c|c|}
\hline $\begin{array}{l}\text { meme } \\
\text { nt }\end{array}$ & & & & & & & & $\begin{array}{l}\text { droits ou } \\
\text { contester un } \\
\text { jugement. Peu } \\
\text { de réponses. }\end{array}$ \\
\hline $\begin{array}{l}\text { Éduca } \\
\text { loi }\end{array}$ & $\begin{array}{l}\text { Page } \\
\text { rattach } \\
\text { ée à } \\
\text { un } \\
\text { organi } \\
\text { sme } \\
\text { sans } \\
\text { but } \\
\text { lucrati } \\
\text { f }\end{array}$ & $\begin{array}{l}\text { Publiqu } \\
\mathrm{e}\end{array}$ & $\begin{array}{l}\text { Plus de } \\
24000\end{array}$ & $\begin{array}{l}\text { Rattaché à ceux } \\
\text { de l'organisme } \\
\text { (aider les } \\
\text { citoyens à mieux } \\
\text { connaître la loi, } \\
\text { leurs droits et } \\
\text { leurs } \\
\text { obligations). }\end{array}$ & $\begin{array}{l}\text { Général } \\
\text { (travail, } \\
\text { logement, } \\
\text { consommation, } \\
\text { etc.) }\end{array}$ & $\begin{array}{l}\text { Oui, par les } \\
\text { gestionnaires de } \\
\text { la page }\end{array}$ & Oui & $\begin{array}{l}\text { Partage des } \\
\text { articles } \\
\text { d'information } \\
\text { juridique du site } \\
\text { de l'organisme } \\
\text { par les } \\
\text { gestionnaires de } \\
\text { la page, } \\
\text { commentaires } \\
\text { des internautes } \\
\text { sous les } \\
\text { articles : } \\
\text { questions, } \\
\text { demandes de } \\
\text { précisions, } \\
\text { questions sur un } \\
\text { autre sujet que } \\
\text { celui de } \\
\text { l'article, } \\
\text { identification } \\
\text { d'« amis » } \\
\text { vivant une } \\
\text { problématique } \\
\text { en lien avec } \\
\text { l'article et } \\
\text { commentaires } \\
\text { de ceux-ci. }\end{array}$ \\
\hline $\begin{array}{l}\text { Mordu } \\
\text { s } \\
\text { d'imm } \\
\text { obilier }\end{array}$ & $\begin{array}{l}\text { Group } \\
\mathrm{e}\end{array}$ & Public & $\begin{array}{l}\text { Plus de } \\
21000\end{array}$ & $\begin{array}{l}\text { Rattaché au } \\
\text { développement } \\
\text { de liens } \\
\text { d'affaires, mais } \\
\text { forte présence } \\
\text { de questions de } \\
\text { nature juridique }\end{array}$ & $\begin{array}{l}\text { Droit du } \\
\text { logement, de } \\
\text { l'immobilier }\end{array}$ & $\begin{array}{l}11 \\
\text { administrateurs } \\
\text { et modérateurs }\end{array}$ & Oui & $\begin{array}{l}\text { Questions de } \\
\text { propriétaires en } \\
\text { droit du } \\
\text { logement } \\
\text { (recours } \\
\text { possibles, } \\
\text { délais, rédaction } \\
\text { de procédures, } \\
\text { demande de } \\
\text { conseils sur une } \\
\text { situation avec } \\
\text { des locataires). }\end{array}$ \\
\hline $\begin{array}{l}\text { Optio } \\
\mathrm{n} \\
\text { conso } \\
\text { mmate } \\
\text { urs }\end{array}$ & $\begin{array}{l}\text { Page } \\
\text { rattach } \\
\text { ée à } \\
\text { un } \\
\text { organi } \\
\text { sme } \\
\text { sans } \\
\text { but } \\
\text { lucrati } \\
\text { f }\end{array}$ & $\begin{array}{l}\text { Publiqu } \\
\mathrm{e}\end{array}$ & Plus de 3000 & $\begin{array}{l}\text { Rattaché à ceux } \\
\text { de l'organisme } \\
\text { (défendre et } \\
\text { protéger les } \\
\text { droits du } \\
\text { consommateur) }\end{array}$ & $\begin{array}{l}\text { Droit de la } \\
\text { consommation }\end{array}$ & $\begin{array}{l}\text { Par les } \\
\text { gestionnaires de } \\
\text { la page }\end{array}$ & Oui & $\begin{array}{l}\text { Partage } \\
\text { d'articles du site } \\
\text { web, ou issus } \\
\text { d'autres sites (y } \\
\text { compris de } \\
\text { médias } \\
\text { d'information) } \\
\text { relatifs à la } \\
\text { consommation. } \\
\text { Quelques } \\
\text { questions } \\
\text { juridiques } \\
\text { ponctuelles des }\end{array}$ \\
\hline
\end{tabular}




\begin{tabular}{|c|c|c|c|c|c|c|c|c|}
\hline & & & & & & & & $\begin{array}{l}\text { internautes, } \\
\text { mais peu } \\
\text { d'interactions. }\end{array}$ \\
\hline $\begin{array}{l}\text { Outra } \\
\text { ge au } \\
\text { tribun } \\
\text { al/ } \\
\text { Conte } \\
\text { mpt of } \\
\text { court }\end{array}$ & Page & $\begin{array}{l}\text { Publiqu } \\
\mathrm{e}\end{array}$ & Environ 1000 & $\begin{array}{l}\text { Page rattachée à } \\
\text { une clinique } \\
\text { juridique par et } \\
\text { pour des } \\
\text { activistes }\end{array}$ & Droit pénal & $\begin{array}{l}\text { Par les } \\
\text { gestionnaires de } \\
\text { la page }\end{array}$ & Non & $\begin{array}{l}\text { Publication de } \\
\text { jugements, de } \\
\text { résumés de } \\
\text { jugements et } \\
\text { d'évènements, } \\
\text { d'information. } \\
\text { Quelques } \\
\text { demandes de } \\
\text { précisions sur } \\
\text { les jugements } \\
\text { dans les } \\
\text { commentaires. }\end{array}$ \\
\hline $\begin{array}{l}\text { Spotte } \\
\text { d } \\
\text { Juridi } \\
\text { que } \\
\text { Provin } \\
\text { ce de } \\
\text { Québe } \\
\text { c }\end{array}$ & Page & $\begin{array}{l}\text { Publiqu } \\
\mathrm{e}\end{array}$ & Environ 50 & $\begin{array}{l}\text { Publication de } \\
\text { questions } \\
\text { juridiques de } \\
\text { manière } \\
\text { anonyme }\end{array}$ & Général & $\begin{array}{l}\text { Par les } \\
\text { gestionnaires de } \\
\text { la page. }\end{array}$ & Non & $\begin{array}{l}\text { Questions } \\
\text { anonymes } \\
\text { publiées à partir } \\
\text { du gestionnaire } \\
\text { de page sur des } \\
\text { thèmes en droit } \\
\text { de la famille } \\
\text { (ex. : pension } \\
\text { alimentaire). } \\
\text { Peu de réponses } \\
\text { aux questions. }\end{array}$ \\
\hline $\begin{array}{l}\text { Supre } \\
\text { me } \\
\text { court } \\
\text { of } \\
\text { Canad } \\
\text { a }\end{array}$ & $\begin{array}{l}\text { Page } \\
\text { officie } \\
\text { lle }\end{array}$ & $\begin{array}{l}\text { Publiqu } \\
\mathrm{e}\end{array}$ & Environ 3000 & $\begin{array}{l}\text { Version } \\
\text { anglophone de } \\
\text { la page sur la } \\
\text { Cour suprême }\end{array}$ & Général & $\begin{array}{l}\text { Par les } \\
\text { gestionnaires de } \\
\text { la page }\end{array}$ & Oui & $\begin{array}{l}\text { Même contenu } \\
\text { que pour sa } \\
\text { version } \\
\text { francophone. }\end{array}$ \\
\hline
\end{tabular}

2. Pages et groupes consacrés au droit qui supposent la participation à un évènement commun

\begin{tabular}{|c|c|c|c|c|c|c|c|c|}
\hline & Type & Ouverture & $\begin{array}{c}\text { Nombre } \\
\text { approximatif } \\
\text { d'internautes }\end{array}$ & $\begin{array}{l}\text { Description } \\
\text { des objectifs } \\
\text { de la page }\end{array}$ & $\begin{array}{l}\text { Domaines } \\
\text { de droits } \\
\text { abordés }\end{array}$ & $\begin{array}{l}\text { Présence de } \\
\text { modération }\end{array}$ & $\begin{array}{l}\text { Activité } \\
\text { récente }\end{array}$ & $\begin{array}{l}\text { Types de } \\
\text { contenus } \\
\text { présents }\end{array}$ \\
\hline $\begin{array}{l}\text { Contestati } \\
\text { on P6 } \\
1 * * * *\end{array}$ & Groupe & Fermé $* * * * *$ & Environ 200 & $\begin{array}{l}\text { Pas de } \\
\text { description, } \\
\text { mais } \\
\text { échanges sur } \\
\text { la } \\
\text { contestation } \\
\text { de } \\
\text { l'arrestation } \\
\text { ayant eu lieu } \\
\text { à une date } \\
\text { précise }\end{array}$ & Pénal & $\begin{array}{l}\text { Neuf } \\
\text { administrate } \\
\text { urs }\end{array}$ & Non & $\begin{array}{l}\text { Questions sur la } \\
\text { procédure pour } \\
\text { déposer une } \\
\text { requête, partage } \\
\text { de jugements en } \\
\text { lien avec la } \\
\text { contestation, } \\
\text { information sur } \\
\text { le } \\
\text { fonctionnement } \\
\text { de la cour, etc. } \\
\text { Partage } \\
\text { d'évènements } \\
\text { ayant lieu hors- } \\
\text { ligne, comme }\end{array}$ \\
\hline
\end{tabular}




\begin{tabular}{|c|c|c|c|c|c|c|c|c|}
\hline & & & & & & & & $\begin{array}{l}\text { des séances } \\
\text { d'information. }\end{array}$ \\
\hline $\begin{array}{l}\text { Contestati } \\
\text { on P6 } \\
2 * * * *\end{array}$ & Groupe & Fermé & Environ 250 & $\begin{array}{l}\text { Protéger le } \\
\text { droit } \\
\text { d'association } \\
\text { et de } \\
\text { rassemblemen } \\
\text { t pacifique }\end{array}$ & Pénal & $\begin{array}{l}\text { Neuf } \\
\text { administrate } \\
\text { urs et } \\
\text { modérateurs }\end{array}$ & Non & $\begin{array}{l}\text { Similaire au } \\
\text { groupe } \\
\text { précédent. }\end{array}$ \\
\hline $\begin{array}{l}\text { Contestati } \\
\text { on de la } \\
\text { contravent } \\
\text { ion } \mathrm{A}^{* * * *}\end{array}$ & Groupe & Fermé & Plus de 150 & $\begin{array}{l}\text { Contester une } \\
\text { contravention } \\
\text { pour } \\
\text { manifestation } \\
\text { illégale à la } \\
\text { suite d'une } \\
\text { arrestation de } \\
\text { masse }\end{array}$ & Pénal & $\begin{array}{l}\text { Quatre } \\
\text { administrate } \\
\text { urs }\end{array}$ & Oui & $\begin{array}{l}\text { Partage de faits } \\
\text { concernant } \\
\text { l'arrestation, } \\
\text { questions sur } \\
\text { comment } \\
\text { présenter une } \\
\text { requête, etc. }\end{array}$ \\
\hline $\begin{array}{l}\text { Recours } \\
\text { collectif } \\
\text { A**** }\end{array}$ & Groupe & Fermé & Environ 250 & $\begin{array}{l}\text { Rassembler } \\
\text { les personnes } \\
\text { arrêtées à une } \\
\text { date précise } \\
\text { pour échanger } \\
\text { sur les recours } \\
\text { juridiques en } \\
\text { cours }\end{array}$ & $\begin{array}{l}\text { Recours } \\
\text { collectif, } \\
\text { contestatio } \\
\mathrm{n} \text { de } \\
\text { contraventi } \\
\text { on (pénal) }\end{array}$ & $\begin{array}{l}\text { Deux } \\
\text { administrate } \\
\text { urs }\end{array}$ & Non & $\begin{array}{l}\text { Vise davantage } \\
\text { à informer à } \\
\text { propos du } \\
\text { recours qu'à } \\
\text { formuler des } \\
\text { besoins } \\
\text { d'information } \\
\text { juridique. }\end{array}$ \\
\hline
\end{tabular}

3. Les pages et groupes qui ne sont pas consacrés au droit, mais où l'on retrouve ponctuellement des contenus d'information juridique

\begin{tabular}{|c|c|c|c|c|c|c|c|c|}
\hline & Type & Ouverture & $\begin{array}{c}\text { Nombre } \\
\text { approximati } \\
\mathbf{f} \\
\text { d'internaut } \\
\text { es }\end{array}$ & $\begin{array}{c}\text { Description } \\
\text { des objectifs } \\
\text { de la page }\end{array}$ & $\begin{array}{l}\text { Domaines de } \\
\text { droits abordés }\end{array}$ & $\begin{array}{l}\text { Présence de } \\
\text { modération }\end{array}$ & $\begin{array}{l}\text { Activ } \\
\text { ité } \\
\text { récen } \\
\text { te }\end{array}$ & $\begin{array}{l}\text { Types de } \\
\text { contenus } \\
\text { présents }\end{array}$ \\
\hline $\begin{array}{l}\text { Chez } \\
\text { Queer } \\
\text { Montreal }\end{array}$ & Groupe & Fermé & $\begin{array}{l}\text { Plus de } \\
10000\end{array}$ & $\begin{array}{l}\text { Partage de } \\
\text { logements et de } \\
\text { colocations } \\
\text { pour les } \\
\text { personnes } \\
\text { queer et alliées. } \\
\text { La description } \\
\text { renvoie à des } \\
\text { ressources } \\
\text { d'information } \\
\text { juridique sur sa } \\
\text { description } \\
\text { pour les } \\
\text { locataires }\end{array}$ & $\begin{array}{l}\text { Droit du } \\
\text { logement }\end{array}$ & $\begin{array}{l}3 \\
\text { administrateurs }\end{array}$ & Oui & $\begin{array}{l}\text { De manière } \\
\text { occasionnelle } \\
\text {, questions en } \\
\text { droit du } \\
\text { logement, } \\
\text { références à } \\
\text { des sites } \\
\text { d'information } \\
\text { juridique, } \\
\text { réponses des } \\
\text { internautes. }\end{array}$ \\
\hline $\begin{array}{l}\text { Immigrati } \\
\text { on } \\
\text { Canada : } \\
\text { étapes et } \\
\text { procédure } \\
\text { s }\end{array}$ & Groupe & Fermé & $\begin{array}{l}\text { Plus de } \\
150000\end{array}$ & $\begin{array}{l}\text { Ressources } \\
\text { pour } \\
\text { comprendre les } \\
\text { différents } \\
\text { programmes } \\
\text { d'immigration } \\
\text { au Canada }\end{array}$ & $\begin{array}{l}\text { Droit de } \\
\text { l'immigration }\end{array}$ & $\begin{array}{l}11 \\
\text { administrateurs } \\
\text { et modérateurs }\end{array}$ & Oui & $\begin{array}{l}\text { Questions } \\
\text { juridiques } \\
\text { très } \\
\text { ponctuelles } \\
\text { (caractère } \\
\text { légal d'une } \\
\text { procédure, } \\
\text { comment }\end{array}$ \\
\hline
\end{tabular}




\begin{tabular}{|c|c|c|c|c|c|c|c|c|}
\hline & & & & & & & & $\begin{array}{l}\text { déposer une } \\
\text { demande de } \\
\text { résidence } \\
\text { permanente). }\end{array}$ \\
\hline $\begin{array}{l}\text { Immigrati } \\
\text { on } \\
\text { Québec }\end{array}$ & Groupe & Fermé & $\begin{array}{l}\text { Plus de } \\
30000\end{array}$ & $\begin{array}{l}\text { Questions et } \\
\text { entraide sur } \\
\text { l'immigration } \\
\text { et l'intégration } \\
\text { au Québec }\end{array}$ & $\begin{array}{l}\text { Droit de } \\
\text { l'immigration }\end{array}$ & $\begin{array}{l}\text { Un } \\
\text { administrateur }\end{array}$ & Oui & Idem. \\
\hline $\begin{array}{l}\text { Logement } \\
\text { s à louer, } \\
\text { Montréal }\end{array}$ & Groupe & Fermé & $\begin{array}{l}\text { Plus de } \\
45000\end{array}$ & $\begin{array}{l}\text { Groupe pour } \\
\text { louer } \\
\text { rapidement un } \\
\text { logement }\end{array}$ & $\begin{array}{l}\text { Droit du } \\
\text { logement }\end{array}$ & $\begin{array}{l}1 \\
\text { administrateur }\end{array}$ & Oui & $\begin{array}{l}\text { Similaire à } \\
\text { d'autres } \\
\text { groupes en } \\
\text { logement : } \\
\text { questions } \\
\text { juridiques } \\
\text { ponctuelles. }\end{array}$ \\
\hline $\begin{array}{l}\text { LOGEME } \\
\text { NTS } \\
\text { MONTRÉ } \\
\text { AL }\end{array}$ & Groupe & Fermé & $\begin{array}{l}\text { Plus de } \\
17000\end{array}$ & $\begin{array}{l}\text { Groupe pour } \\
\text { partager des } \\
\text { logements à } \\
\text { louer }\end{array}$ & $\begin{array}{l}\text { Droit du } \\
\text { logement }\end{array}$ & $\begin{array}{l}3 \\
\text { administrateurs }\end{array}$ & Oui & $\begin{array}{l}\text { Similaire à } \\
\text { d'autres } \\
\text { groupes en } \\
\text { logement : } \\
\text { questions } \\
\text { juridiques } \\
\text { ponctuelles. }\end{array}$ \\
\hline $\begin{array}{l}\text { PVTistes } \\
\text { à } \\
\text { Montréal }\end{array}$ & Groupe & Public & $\begin{array}{l}\text { Plus de } \\
30000\end{array}$ & $\begin{array}{l}\text { Entraide entre } \\
\text { personnes } \\
\text { vivant à } \\
\text { Montréal et } \\
\text { ayant un permis } \\
\text { de travail (ou } \\
\text { autres } \\
\text { personnes } \\
\text { immigrantes) }\end{array}$ & $\begin{array}{l}\text { Droit du } \\
\text { logement, de } \\
\text { l'immigration. }\end{array}$ & $\begin{array}{l}9 \\
\text { administrateurs } \\
\text { et modérateurs }\end{array}$ & Oui & $\begin{array}{l}\text { Questions } \\
\text { très } \\
\text { occasionnelle } \\
\text { s en rapport } \\
\text { avec des } \\
\text { procédures } \\
\text { judiciaires en } \\
\text { immigration. }\end{array}$ \\
\hline $\begin{array}{l}\text { Vélo } \\
\text { d'hiver }\end{array}$ & Groupe & Public & $\begin{array}{l}\text { Plus de } \\
8000\end{array}$ & $\begin{array}{l}\text { Partage de } \\
\text { récits et } \\
\text { d'information } \\
\text { sur le vélo } \\
\text { d'hiver }\end{array}$ & $\begin{array}{l}\text { Pénal } \\
\text { (contraventions) }\end{array}$ & $\begin{array}{l}11 \\
\text { administrateurs }\end{array}$ & Oui & $\begin{array}{l}\text { Demandes } \\
\text { d'information } \\
\text { sur les } \\
\text { manières de } \\
\text { contester une } \\
\text { contravention } \\
\text { et les } \\
\text { arguments } \\
\text { importants en } \\
\text { justice, } \\
\text { partage de } \\
\text { récits par } \\
\text { rapport à une } \\
\text { contravention }\end{array}$ \\
\hline
\end{tabular}

*Dans les 30 derniers jours

**Tout le monde peut voir le groupe, ses membres ainsi que leurs leurs publications

***Qui est rattachée à l'institution qu'elle représente, par opposition à une page non officielle

$* * * *$ Noms exacts altérés

***** Tout le monde peut voir le groupe (par opposition à un groupe secret), mais il faut joindre le groupe afin d'voir les discussions, de publier et de commenter les publications des autres. 